Ersch. in: Politische Vierteljahresschrift 50 (2009), 3, S. 539-576

\title{
Vom ungeliebten Störenfried zum akzeptierten Paradigma? Zum Stand der (Neuen) Politischen Ökonomie in Deutschland
}

\author{
Katharina Holzinger
}

Zusammenfassung: Der Beitrag präsentiert den Stand der ökonomischen Analyse von Politik in Deutschland. Nach einer kurzen Skizze des Grundmodells, der Gegenstandsbereiche und des analytischen Instrumentariums wird der Forschungsstand in den einzelnen Teildisziplinen der Politikwissenschaft dargestellt. Dabei wird jeweils der deutsche Beitrag im Vergleich zum internationalen Stand beschrieben. In Deutschland wurden die ökonomischen Ansätze zunächst zögerlich aufgenommen. In jüngerer Zeit finden sich jedoch im Bereich der Wahl- und Parteienforschung, der Analyse der EU und der Theorie der Veto-Spieler viele Beiträge aus dieser Perspektive.

Schlagwörter: Politische Ökonomie $\cdot$ Neue Politische Ökonomie $\cdot$ Ökonomische Theorien der Politik $\cdot$ Rational choice

\begin{abstract}
This contribution presents the German state of the art of economic approaches to politics. First, the basic model, the research areas, and the analytical tools used by these approaches are briefly introduced. Second, for each sub-field of political science, the German contribution is presented in relation to the international state of the art. While German scholars hesitated to engage in public choice and positive political theory in the beginning, we nowadays find many contributions from this perspective in the fields of electoral studies, the European Union, and veto player analysis.
\end{abstract}

Keywords: Political Economy $\cdot$ Positive Political Theory $\cdot$ Public Choice $\cdot$ Collective Choice 


\section{Einleitung}

Den Stand der Forschung zur Politischen Ökonomie (PÖ) in Deutschland in Bezug zu setzen zu den internationalen Entwicklungen ist vor allem deshalb schwierig, weil der Begriff PÖ äußerst unscharf ist. Zum einen existiert er seit 300 Jahren und hat seither viele Wandlungen erfahren. Zum anderen werden damit auch heute noch verschiedene Forschungsrichtungen und Ansätze bezeichnet, die alle Teilgebiete der Politikwissenschaft betreffen und verschiedene Gegenstandsbereiche untersuchen.

Nach einem kurzen Überblick über die verschiedenen Varianten der PÖ skizziere ich in Abschnitt 2 das Grundmodell, die Gegenstandsbereiche und das analytische Instrumentarium der ökonomischen Theorie der Politik. In Abschnitt 3 stelle ich den Forschungsstand der politisch-ökonomischen Ansätze in den Teildisziplinen der Politikwissenschaft dar. Dabei wird jeweils zunächst der internationale Stand kurz vorgestellt und anschließend der deutsche Beitrag präsentiert. Den Zeithorizont bilden die vergangenen 50 Jahre. In Abschnitt 4 werden die institutionelle Verankerung der PÖ und die wichtigsten Publikationsorgane beschrieben.

\section{Varianten, theoretisches Grundmodell, Gegenstände und analytisches Instrumentarium}

\subsection{Varianten der Politischen Ökonomie}

Eine allgemeine Definition von PÖ ist kaum möglich. Der Begriff entstand Anfang des 17. Jahrhunderts und bezog sich ursprünglich sowohl auf die Wirtschaftspolitik eines Staates als auch auf die theoretische Reflexion darüber. Die PÖ als wissenschaftliche Disziplin begann mit der Wirtschaftsliteratur in der Phase des Merkantilismus. Als Reaktion auf den Merkantilismus entstand die klassische PÖ (Adam Smith, John Stuart Mill, David Ricardo). Wesentlich war das Plädoyer für die wirtschaftspolitische Zurückhaltung des Staates. In der Neoklassik spielte die PÖ dann keine Rolle mehr. In der marxistischen $P \ddot{O}$ (auch Politökonomie) wird das politische System als eine sich historisch wandelnde Form der Herrschaft über die Produktionsmittel begriffen. Der Staat ist zentrale Planungsinstanz, die eine an den Bedürfnissen der Bevölkerung orientierte Versorgung mit Gütern und Dienstleistungen vorzunehmen hat. Die neomarxistische PÖ und der Reformsozialismus („Neue Linke“, „Radical Economics“) betonten meist die Ungleichheit der Einkommens- und Vermögensverteilung im Kapitalismus.

Aufgrund dieser Geschichte hatte der Begriff PÖ eine anti-kapitalistische Konnotation, wogegen sich die Vertreter der Neuen Politischen Ökonomie (NPÖ) mit dem Wort „neu“ abzugrenzen suchten. Dieser seit den 1950er Jahren entstandene Ansatz bedient sich des Instrumentariums der modernen Wirtschaftstheorie. Ausgehend vom ökonomischen Handlungsmodell analysiert die NPÖ einerseits politische Prozesse und Institutionen an sich, andererseits auch deren Einfluss auf das Wirtschaftsgeschehen.

Daneben werden heute einige nicht der NPÖ im engen Sinn zuzurechnende Ansätze der Wirtschafts- und Politikwissenschaft, deren Gemeinsamkeit im Interesse am Überschneidungsbereich von Politik und Wirtschaft liegt, als PÖ (political economy) be- 
zeichnet. Dies umfasst Analysen von klassischen Feldern der nationalen Wirtschaftspolitik, also etwa der makroökonomischen Steuerung, der Steuer- und Währungspolitik, der Verschuldung, der industriellen Beziehungen und der sozial- und wohlfahrtsstaatlichen Politik, aber auch die als Internationale PÖ zusammengefassten Felder der Außenwirtschaftspolitik (Handel und Finanzmärkte), der Globalisierungsforschung und der Entwicklungspolitik. Auch vergleichende Analysen der Wirtschaftssysteme, etwa die Forschung zu den Typen von Wohlfahrtsstaaten (Esping-Andersen 1990) oder zu den Varieties of Capitalism (Hall/Soskice 2001), werden der PÖ zugerechnet.

In der heutigen Begriffsverwendung von PÖ sind folglich zwei Ansätze zu unterscheiden, nämlich

1. Studien, die ökonomische Annahmen, Modelle und Methoden zur Analyse politikwissenschaftlicher Gegenstände verwenden, und

2. Studien, die Untersuchungsgegenstände aus dem Überschneidungsbereich Politik und Wirtschaft erforschen.

Zugespitzt könnte man sagen, dass beim ersten Typ Gegenstände der Politikwissenschaft mit einem der Ökonomik entlehnten Instrumentarium, beim zweiten Typ ökonomische Gegenstände mit politikwissenschaftlichen Ansätzen behandelt werden. Das trifft insofern nicht ganz, als es sich bei den betrachteten „ökonomischen Gegenständen“ um Politiken, also zugleich auch um politikwissenschaftliche Gegenstände handelt. Die analytische Trennung kann außerdem in der Praxis nicht immer aufrechterhalten werden, da Studien vom zweiten Typ häufig zugleich auch dem ersten Typ zuzuordnen sind, weil sie ihren wirtschaftspolitischen Gegenstand mit Ansätzen erforschen, die auf das ökonomische Handlungsmodell rekurrieren.

Dem Forschungsprofil der Autorin entsprechend erfolgt hier eine Konzentration auf den erstgenannten Ansatz der PÖ. Wegen der Überschneidung werden aber auch Beiträge zur Analyse von Wirtschaftspolitik und internationaler PÖ angesprochen, soweit sie mit dem ökonomischen Instrumentarium arbeiten. Dies schließt z. B. neo-marxistische, neo-gramscianische und soziologische PÖ aus.

\subsection{Theoretisches Grundmodell}

Ökonomische Theorien der Politik verwenden das Handlungsmodell und das analytische Instrumentarium der Wirtschaftswissenschaft zur Erklärung politischer Strukturen und Prozesse. Allen Arbeiten dieser Ausrichtung gemeinsam ist ein mikrofundierter handlungstheoretischer Ansatz, der Rationalität der Akteure voraussetzt. Ausgangspunkt ist der methodologische Individualismus: Soziale Prozesse werden auf der Basis von Annahmen über das Verhalten einzelner Akteure erklärt, wobei die Präferenzen kollektiver Akteure (z.B. Organisationen) oft, aber nicht immer auf individuelle Präferenzen rückgeführt werden (vgl. Coleman 1994). Rationalität bedeutet dabei, Entscheidungen an den Konsequenzen des Handelns zu orientierten. Es bedeutet nicht, dass die Handlungsmotivation eigennützig sein muss. In den neueren Arbeiten wird außerdem die traditionelle Annahme des vollständig informierten Akteurs aufgegeben und von begrenzter Information der Akteure ausgegangen (beginnend mit Simon 1957).

Nach dem ökonomischen Handlungsmodell reagieren Akteure systematisch auf Anreize, die sich (a) aus ihren Präferenzen und (b) aus (ihrer Wahrnehmung von) extern 
gegebenen Handlungsbeschränkungen ergeben. Sie wählen aus den ihnen zur Verfügung stehenden Handlungsalternativen diejenige aus, von der sie sich den höchsten Nutzen versprechen. Dies setzt voraus, dass die Akteure in der Lage sind, ihre Ziele gemäß einer Präferenzordnung konsistent zu ordnen und die Handlungsalternativen nach ihren erwarteten Wirkungen zu bewerten. Die Präferenzordnung soll bei Entscheidungen unter Unsicherheit zudem in Einklang mit den Überzeugungen über die Zustände der Welt und den „Vorschriften“ der Erwartungsnutzentheorie stehen. Da Präferenzen nur schwer beobachtbar und messbar sind und zudem als relativ stabil angenommen werden, werden in den ökonomischen Modellen Verhaltensänderungen - soweit möglich - durch Änderungen in den Handlungsbeschränkungen oder Informationsständen erklärt. Häufig modellierte Beschränkungen sind das Einkommen, die Zeit (Goodin et al. 2008) und andere Handlungsressourcen sowie alle sozialen Institutionen. Theorien, die diesen Annahmen folgen, werden inzwischen in den Sozialwissenschaften allgemein dem Rational-choice-Ansatz (RC) zugeordnet.

\subsection{Gegenstandsbereiche}

$\mathrm{Zu}$ den ökonomischen Theorien der Politik im weiteren Sinne zählen einige ökonomische Vorläufer-Theorien, in denen in der ersten Hälfte des 20. Jahrhunderts die Bedingungen des Marktversagens herausgearbeitet wurden (Abschnitt 2.3.1). Hieraus entwickelte sich nach dem zweiten Weltkrieg die ökonomische Theorie der Politik im engeren Sinne, die zu Beginn folgerichtig als ,,theory of non-market decision-making " bezeichnet wurde (Abschnitt 2.3.2). Parallel entwickelten sich im Rahmen einer oft als ,ökonomischer Imperialismus" gebrandmarkten Bewegung weitere Ansätze, die das ökonomische Handlungsmodell auf nicht-ökonomische Gegenstände anwendeten (z. B. Becker 1976). Für die Politikwissenschaft einschlägig sind die ökonomische Theorie des Rechts und die Institutionenökonomie (Abschnitt 2.3.3).

\subsubsection{Tauschtheorie, Wohlfahrtsökonomie und Kollektivgüter}

Die ökonomischen Ansätze lassen sich auf die klassischen Tauschtheorien zurückführen (ausführlich Maloy 2008; Wiesenthal 1987). Diese befassten sich mit der Entstehung von Märkten als geordneten Tauschbeziehungen von Individuen (vgl. Coleman 1994). Märkte sind das Ergebnis individueller Wahlhandlungen, die auf der Basis subjektiver Nutzenkalküle getroffen werden. Unter Bedingungen vollkommener Konkurrenz führt dezentrale Koordination über den Preismechanismus zu gesellschaftlich optimalen Ergebnissen - so das „erste fundamentale Theorem“ der Wohlfahrtsökonomie. Die Verfolgung des Eigeninteresses dient somit unbeabsichtigt den Interessen der Gesellschaft.

Dass eine Tauschwirtschaft auch bei vollständiger Konkurrenz ohne staatliche Eingriffe keineswegs zu optimalen Ergebnissen führen muss, zeigte die wohlfahrtsökonomische Theorie des Marktversagens. Betont wurden in diesem Zusammenhang die Notwendigkeit einer staatlichen Wettbewerbspolitik, die Produktionsbedingungen „,natürlicher Monopole“" sowie die Existenz von Externalitäten und Kollektivgütern, deren zwei grundlegende Eigenschaften Nicht-Rivalität im Konsum und Nicht-Ausschließ- 
barkeit vom Konsum bei marktlicher Bereitstellung zu Unterversorgung oder zu Übernutzung der betroffenen Güter führe.

\subsubsection{Neue Politische Ökonomie (NPÖ, public choice)}

Diese ökonomischen Begründungen einer Staatstätigkeit über den liberalen „Nachtwächter" hinaus weckte das Interesse der Ökonomen an der politischen Bereitstellung von Gütern und damit an politischen Entscheidungsprozessen. Die NPÖ umfasst die Theorie der Präferenzaggregation, die Theorie der Verfassung, Theorien der Demokratie, der Interessengruppen, der Bürokratie und der Autokratie (Mueller 2001, 2003).

1. Gegenstand der Theorie der Präferenzaggregation (collective choice) ist die Frage, wie individuelle Präferenzen möglichst frei von Verfälschungen in kollektive Entscheidungen umgesetzt werden können. Die Unmöglichkeit einer solchen sozialen Wohlfahrtsfunktion wurde von Arrow (1951) nachgewiesen. Wenngleich die Relevanz des Theorems strittig ist, konnte es bisher nicht widerlegt werden.

2. Die Theorie der Verfassung (konstitutionelle PÖ) ist eine normative Spielart der NPÖ, die sich mit der Begründung von Verfassungen (in den neuen Vertragstheorien) und ihren grundlegenden Regeln auseinandersetzt, etwa der von Buchanan/ Tullock (1962) vorgeschlagenen Einstimmigkeitsregel.

3. Die ökonomische Theorie der Demokratie zielt auf die Erklärung des Verhaltens von Wählern, Parteien und Regierungen. Das Medianwählermodell von Black (1958) und die Theorie der Parteienkonkurrenz von Downs (1957) standen am Beginn dieses Teilgebiets.

4. Die PÖ der Interessengruppen befasst sich mit der Organisierbarkeit von Interessengruppen und deren Einfluss auf politische Entscheidungen. Sie wurde begründet durch Olsons „Logik des kollektiven Handelns“ (1965). In der Literatur zum rentseeking (Krueger 1974) wurde der Einfluss von organisierten Interessen auf die Politik untersucht.

5. Grundlage der ökonomischen Theorien der Bürokratie ist die Auffassung, dass Verwaltungsbeamte nicht per se Diener des Allgemeinwohls sind, sondern dass auch sie eigennützige Ziele wie Einkommen oder Bequemlichkeit verfolgen (Downs 1967). Am einflussreichsten wurde das Modell von Niskanen (1971), der das Ziel Budgetmaximierung unterstellt und ineffiziente Überproduktion durch Bürokratien vorhersagt.

\subsubsection{Institutionenökonomie und Ökonomie des Rechts}

Mit der Entstehung, Funktion und Wirkung von Institutionen, Verträgen und Recht befassen sich die Institutionenökonomie und die Ökonomie des Rechts (Behrens 1986; Pies 1993). Ausgangspunkt ist die Annahme unvollständiger Information. In diesem Fall können Märkte nicht perfekt funktionieren und Hierarchie, Delegation und Organisationen treten an ihre Stelle. 
Die Principal-agent-Theorie beschäftigt sich mit Delegations-Beziehungen zwischen Auftraggebern und Auftragnehmern (z. B. Arzt-Patient, Chef-Mitarbeiter, Wähler-Regierung). Dabei geht es um zwei Probleme: (1) Wie kann der Auftraggeber den Auftragnehmer motivieren, in seinem Interesse zu handeln und wie kann er ihn kontrollieren, wenn der Auftragnehmer einen Informationsvorsprung hat? (2) In einer Welt asymmetrischer Information und ungewisser Zukunft bleiben Verträge notwendig unvollständig, da es unmöglich ist, alle Kontingenzen vorherzusehen. Welche Anreize können eingesetzt werden, um die Probleme des moral hazard (opportunistisches Verhalten) und der adverse selection (die falschen Agenten werden angezogen) zu vermeiden?

Während in der traditionellen Wirtschaftstheorie Institutionen meist als exogene Faktoren behandelt wurden, erklärt die Institutionenökonomie die Wahl zwischen alternativen Institutionen. Grundlegend sind die Theorie der Transaktionskosten von Coase (1960) und deren Weiterentwicklung durch Williamson (1985). Transaktionskosten sind Kosten der Benutzung des Preismechanismus oder anderer Steuerungsmechanismen, die zu Wohlfahrtsverlusten führen. Die zentrale Hypothese ist, dass alternative institutionelle Lösungen zum Zweck der Einsparung von Transaktionskosten entstehen.

\subsection{Analytisches Instrumentarium der PÖ}

Ein wesentliches Merkmal der ökonomischen Theorien der Politik ist, dass sie die formal präzisen Instrumente der Mikroökonomie, der Entscheidungstheorie und der Spieltheorie einsetzen. Zu diesen Werkzeugen gehörten zunächst vor allem die Erwartungsnutzentheorie und die klassische mikroökonomische Marginalanalyse (Olson 1965; Niskanen 1971; Williamson 1985). Aber auch räumliche Modelle der Politik finden sich von Beginn (Black 1958; Downs 1957).

Zum wichtigsten Hilfsmittel entwickelte sich die Spieltheorie, und zwar sowohl in der strategischen als auch in der kooperativen Form. Anwendungen auf politikwissenschaftliche Gegenstände bedienten sich anfangs bevorzugt der kooperativen Spieltheorie: Die Koalitionstheorie und die Machtindizes wurden in den 1950er Jahren entwickelt (z.B. Riker/Shapley 1968; Mann/Shapley 1964) und wurden zum Standardinstrument politikwissenschaftlicher Analyse aus der Perspektive der „Positiven Politischen Theorie“ (Riker 1963). Auch die kooperativen Verhandlungsmodelle (Nash 1950a) fassten $\mathrm{Fuß}$ in der PÖ und spielen heute wieder eine größere Rolle in der Analyse der internationalen Beziehungen (z. B. Fearon 1995). Auf der Koalitionstheorie und den räumlichen Politikmodellen basiert schließlich die Theorie der Vetospieler von Tsebelis (2000).

Da es bei den Gegenständen der PÖ oft um die strategische Interaktion weniger Akteure geht, setzte sich die nicht-kooperative Spieltheorie schnell als Instrumentarium zur Formalisierung der theoretischen Modelle durch. Die Spieltheorie schien angemessener als das klassische mikroökonomische Werkzeug, das für Marktprozesse mit vielen Akteuren entwickelt worden war (Amadae/Bueno de Mesquita 1999). Die Spieltheorie selbst machte zwischen 1950 und 1980 große Fortschritte: von einfachen Lösungen für Einmalspiele unter vollständiger Information, etwa dem Nash-Gleichgewicht (von Neumann/Morgenstern 1944; Nash 1950b) bis zu elaborierten Gleichgewichtskonzepten für wiederholte Spiele und Spiele unter unvollständiger Information (Überblick bei Fuden- 
berg/Tirole 1991). Inzwischen liegen auch spezifisch auf politikwissenschaftliche Anwendungen ausgerichtete Lehrbücher der Spieltheorie vor (Shubik 1982; Morrow 1994; McCarthy/Meirowitz 2007).

\section{Politische Ökonomie in den politikwissenschaftlichen Teildisziplinen}

\subsection{Politische Theorie}

\subsubsection{Der internationale Stand}

Im Bereich der Politischen Theorie lassen sich vier Gebiete identifizieren, in denen es zu Debatten und Weiterentwicklungen der PÖ kam: die konstitutionelle PÖ, die Positive Political Theory, der RC-Ansatz und das ökonomische Akteursmodell.

Die konstitutionelle PÖ wurde vor allem von ihrem Begründer Buchanan und seinen Ko-Autoren weiterverfolgt. In „The Limits of Liberty“ (1975) behandelte er das Problem des Zustandekommens einer Rechtsordnung und die Rolle des Staates als Rechtsdurchsetzer und Produzent öffentlicher Güter. Indem er die Umverteilung nicht als primäre Staatsaufgabe definiert, nimmt er den Dialog auf mit den neueren Vertragstheorien und v. a. mit ihrer bekanntesten Version, der Rawls'schen Theorie der Gerechtigkeit (1971). Brennan/Buchanan (1980) legten ein Modell der Regierung als Einnahmen maximierender Leviathan vor, das sie zu der Folgerung führte, dass Steuern, Staatsverschuldung und Geldmengensteuerung konstitutionell beschränkt werden sollten. In „The Reason of Rules“ (1985) plädieren sie für die positive und normative Analyse von Verfassungsinstitutionen mit ökonomischen Mitteln, die von der Frage geleitet sein sollte, was die besten Regeln für eine Gesellschaft sind. Die Autoren sehen Regeln als Substitut für Moral.

Riker (1963) prägte den Begriff der Positive Political Theory für ein Verständnis von Politischer Theorie, das auf positive (im Gegensatz zu normativer) Analyse, Formalisierung, Gesetze und Vorhersagen setzt. Er sah die Spieltheorie und die räumlichen Modelle als geeignete Ansatzpunkte für ein solches Unternehmen. Diese Werkzeuge wurden in den Lehrbüchern von Ordeshook $(1986,1992)$ und in Alt/Shepsle (1990) einem breiteren Publikum zugänglich gemacht. In Deutschland hat der Terminus allerdings kaum Widerhall gefunden. Die amerikanischen Beiträge zur Positive Political Theory, vor allem aus der Rochester School (Amadae/Bueno de Mesquita 1999), werden im Abschnitt zur Teildisziplin Politische Systemforschung behandelt.

Ausgelöst durch die Publikation von „Pathologies of Rational Choice Theory“ (Green/Shapiro 1994; dt. 1999) wurde die bisher größte Theorie-Debatte zum RC-Ansatz in der angelsächsischen Politikwissenschaft geführt. Green und Shapiro warfen dem Ansatz vor, dass die auf seiner Grundlage gewonnenen abstrakten Theorien der empirischen Überprüfung unzugänglich seien und dass im Rahmen des Ansatzes ,very little has been learned [...] about politics“ (1994: x). Anhand einiger Beispiele demonstrieren sie (vermeintliche) Fehlschläge von RC-Erklärungen: das Paradox des Wählens (unter der Annahme von Rationalität kann Wahlbeteiligung nicht erklärt werden), die Tragödie der Allmende (empirisch lässt sich beobachten, dass sehr wohl Beiträge zu 
öffentlichen Gütern geleistet werden), die Instabilität demokratischer Institutionen (Vorhersage zyklischer Mehrheiten, für die sich aber keine empirische Evidenz finde) und räumliche Modelle zur Vorhersage von Wahlergebnissen (ungeeignet zur Prognose tatsächlichen Kandidatenverhaltens). Die nachfolgende Debatte wurde v.a. in der Zeitschrift Critical Review geführt (Friedman 1996). Drei kontroverse Gegenstände kristallisierten sich heraus: Sind die Einsichten, die RC-basierte Theorie erbringen kann, überhaupt von Bedeutung? Was sind die Aufgaben einer politischen Wissenschaft? Ist das Modell des rationalen Akteurs angemessen? Zwei Beiträge zu dieser Debatte stammen von deutschen Autoren (Diermeier 1996; Lohmann 1996), die allerdings in den USA wissenschaftlich sozialisiert wurden und dort arbeiten.

Das Handlungsmodell der ökonomischen Theorie hat stets Kritik hervorgerufen. Während es in viele Arbeiten unverändert als Annahme eingeht (als normative Vorstellung oder als empirischer Grenzfall), ist der Glaube an seine Korrektheit als empirische Beschreibung auch unter Ökonomen nicht mehr weit verbreitet. Seit den 1970er Jahren wurden umfangreiche experimentelle Forschungen durchgeführt, die das Modell von zwei Seiten untergraben: der kognitiven Kapazität und der volitiv-normativen Zielsetzungen (vgl. Frey 1990).

Erstens zeigte sich, dass in bestimmten Fällen die Voraussetzungen für rationales Handeln systematisch nicht erfüllt sind. Dies wurde zuerst empirisch nachgewiesen von Kahnemann et al. (1982), die zeigen konnten, dass Risiken von Individuen systematisch anders behandelt werden, als dies die Erwartungsnutzentheorie vorhersagt. Eine andere Weiterentwicklung in diesem Bereich geht wesentlich auf Selten zurück, der sich von der Annahme vollständiger Rationalität abwandte und eine experimentell basierte Theorie des Lernens entwickelte (Selten et al. 2005). Zweitens zeigte die experimentelle ökonomische, psychologische und zunehmend auch die neurowissenschaftliche Forschung, dass Entscheidungen von Individuen häufig durch Vorstellungen von Fairness und Ungleichheitsaversion (z. B. Güth et al. 1982; Güth et al. 2006; Ockenfels 1999) sowie von Altruismus und Kooperation (z. B. Fehr/Gächter 2002; Fehr et al. 2003) bestimmt werden, was Veränderungsvorschläge für das zugrunde zu legende Akteursmodells nach sich zog, die von Vertretern des RC-Ansatzes bis heute kontrovers diskutiert werden.

\subsubsection{Der Stand in Deutschland}

Die Wahrnehmung des RC-Ansatzes in der deutschen Politischen Theorie begann ab Ende der 1970er Jahre mit ersten Veröffentlichungen (Kern 1979; Kern/Müller 1986; Zintl 1981, 1983, 1984). Eine Gruppe von Forschern institutionalisierte sich allerdings erst 1993 mit der Gründung des Arbeitskreises Handlungs- und Entscheidungstheorie der DVPW. Dieser interdisziplinäre Kreis begegnete sich auch in anderen, ökonomischen, philosophischen oder soziologischen Kontexten, z. B. im Rahmen des Jahrbuchs für Neue Politische Ökonomie oder der Theorie-Zeitschrift Analyse \& Kritik, wo häufig nationale und internationale Vertreter der NPÖ aus diesen Disziplinen publizieren.

Die frühen Bände des Arbeitskreises zeigen, dass die Hauptthemen sich zunächst um Grundsatzdiskussionen zum RC-Ansatz ranken: Es geht um den homo oecomomicus, das Rationalitätspostulat (z. B. Druwe/Kunz 1994, 1996) und um die sogenannten Ano- 
malien (Druwe/Kunz 1998), etwa das Risikoverhalten oder das Wählerparadox. Die Diskussionen werden nicht anders als und auf dem gleichen Niveau geführt wie in der amerikanischen RC-Kontroverse. Im Gegensatz zur amerikanischen Diskussion spielt allerdings die wissenschaftstheoretische Verortung des Ansatzes eine große Rolle. Hierfür stehen vor allem die Arbeiten von Kunz (1996, 1997, 2004). Prominent sind außerdem Ansätze aus der konstitutionellen Theorie (Zintl 1983, 1986, 2000). Zimmerling (2005) befasst sich aus theoretischer Perspektive mit Macht und Einfluss in der Politik.

Als Pionier der Positiven Politischen Theorie in Deutschland trug Pappi schon früh zur Koalitionstheorie bei (z. B. Pappi/Melbeck 1984; Kappelhoff/Pappi 1984) und arbeitet auch heute noch in diesem Bereich, wobei seine Anwendungsgebiete die EU und die deutschen Bundesländer sind (z. B. Linhart/Pappi/Schmitt 2008; Linhart/Pappi 2009; Pappi/Becker/Herzog 2005; Thurner/Pappi 2009). Seine Arbeiten zur quantitativen Netzwerkanalyse (1987) lassen sich zwar als Teil einer Positiven Politischen Theorie verstehen, sind aber nicht im eigentlichen Sinne der PÖ zuzuordnen. Dagegen gehören Pappis Arbeiten zur Theorie des Wählerverhaltens und den Wirkungen von Wahlsystemen durchaus zum Spektrum der ökonomischen Theorien der Demokratie (z. B. Pappi 1973; Pappi/Shikano 2007), wenn hier auch die Überschneidung zum Behaviourismus deutlich wird.

Eine breitere Wahrnehmung und Akzeptanz in der deutschen Politikwissenschaft hat allerdings die Adaption des RC-Ansatzes durch den akteurzentrierten Institutionalismus von Mayntz und Scharpf erfahren (Mayntz/Scharpf 1995; Scharpf 2000). Dabei werden die Mikrofundierung und das Handlungsmodell des RC-Ansatzes übernommen, der Aspekt der Institutionen wird jedoch stärker betont. Auch eine eigensüchtige Akteursmotivation wird zugunsten von vielschichtigeren Interaktionsorientierungen zurückgewiesen (Scharpf 2000) und die Bedeutung der Fiktion kollektiver Akteure betont (Zintl 1994). Die Formalisierung in mathematischen Modellen und der rigorose quantitative empirische Test stehen nicht im Vordergrund. In der Praxis des akteurzentrierten Institutionalismus dominieren Fall-Rekonstruktionen mittels verhandlungs- und spieltheoretischer Modelle (Benz et al. 1992; Scharpf 1993, 1997; Genschel/Plümper 1997; Ganghof/Manow 2005; Holzinger 2008).

\subsection{Politische Systemforschung}

\subsubsection{Der internationale Stand}

Alle in den 1950er Jahren begründeten Teilbereiche der NPÖ wurden im Laufe der folgenden Jahrzehnte weiterentwickelt und ausdifferenziert. Das Grundmodell der Theorie der Demokratie von Downs (1957) hat besonders durch die Rochester School eine Fülle von Modifikationen, Erweiterungen und Anwendungen erfahren. So wurden z.B. Mehrparteien-Modelle entwickelt und die Möglichkeiten der Koalitionsbildung untersucht. Riker (1963) stellte der Hypothese der Stimmenmaximierung sein Theorem der minimum winning coalitions gegenüber, wonach immer nur so viele Koalitionspartner sich zusammenschließen (bzw. so viele Stimmen angestrebt werden), wie für die notwendige Mehrheit nötig sind (vgl. Grofman 2004a; Amadae/Bueno de Mesquita 1999). 
Auch die bei Downs vernachlässigte Frage der ideologischen Ziele von Parteien wurde in Modelle des Regierungsverhaltens aufgenommen, die annehmen, dass eine Regierung ihren ,ideologischen Nutzen“ unter der Restriktion der Wiederwahl maximiert (Frey 1981). Auf dieser Basis wurden Modelle der politisch-ökonomischen Interdependenz entwickelt. Der Regierung wird unterstellt, dass sie zur Sicherung ihrer Wiederwahl bewusst „politische Konjunkturzyklen“ erzeugt (Frey 1981; Pappi/Shikano 2007). Schließlich wurde gezeigt, dass Downs' empirisch unzutreffende Vorhersage der Konvergenz der Parteien auf impliziten Modellannahmen beruht, bei deren realitätsgerechter Lockerung das Resultat der vollen Konvergenz verschwindet (Grofman 2004b).

Die räumlichen Modelle von Black und Downs wurden mehrdimensional weiterentwickelt (McKelvey 1976; McKelvey/Schofield 1986), was zum Chaos-Theorem führte: Nur unter sehr restriktiven Annahmen sind bei mehrdimensionalen Entscheidungen stabile Politikergebnisse möglich. Da wir in der Empirie aber stabile Ergebnisse beobachten, wurde nach Erklärungen dafür gesucht. Eine Lösung präsentierte die Literatur zum Agenda-setting (Riker 1993), eine andere die Literatur zu strukturinduzierten Gleichgewichten, etwa durch bestimmte Abstimmungsregeln oder die Rolle von Parlamentsausschüssen (Shepsle 1979; Shepsle/Weingast 1984).

Downs' Analyse der Wählerpartizipation hatte ein Problem sichtbar gemacht, für das zunächst keine befriedigende Lösung gefunden werden konnte: Ein rationaler Wähler würde nicht zur Wahl gehen, denn die Informationskosten der politischen Beteiligung sind extrem hoch, die Chancen eines Wählers, den Wahlausgang mit seiner Stimme in seinem Sinne zu beeinflussen, dagegen verschwindend gering. Für dieses Problem wurde eine Reihe von Lösungen vorgeschlagen (z.B. das Einmodellieren moralischer Präferenzen oder die Idee des expressiven Handelns in Niedrigkostensituationen (Brennan/ Hamlin 2000; Brennan/Lomaski 1993; Kliemt 1984; Zintl 1989). In Konkurrenz zu den Parteiidentifikations-Hypothesen der Michigan School formulierten RC-Theoretiker eine Theorie des retrospektiven Wählens, wonach Wähler die vergangene Leistung der Amtsinhaber bewerten (Fiorina 1976; Ferejohn 1986).

Einige der spezielleren Behauptungen Olsons (1965) zum kollektiven Handeln können mittlerweile als widerlegt gelten. Ob die Kollektivgutproblematik überwunden werden kann, hängt meist weniger von der Gruppengröße ab als von anderen Faktoren wie Eigenschaften des fraglichen Kollektivgutes oder Erreichbarkeit und Kontrollmöglichkeit der Gruppenmitglieder (Ostrom 1990; Holzinger 2008). Auch die allgemeine Gültigkeit der Trittbrettfahrerproblematik ist mittlerweile infrage gestellt. Selbst wenn strikt nutzenmaximierende Akteure unterstellt werden, ist bei wiederholtem Auftreten derselben Situation mit Kooperation zu rechnen, wie die Spieltheorie anhand des unendlich wiederholten Gefangenendilemmas gezeigt hat (Taylor 1987; Axelrod 1984). Wie Kooperationsprobleme bei der Bereitstellung von Allmendegütern gelöst werden können, hat Ostrom in vielen Fallstudien und Analysen institutioneller Vorkehrungen untersucht (z.B. 1990, 1998). Auch Experimente haben ergeben, dass in Trittbrettfahrersituationen durchaus kooperatives Verhalten gezeigt wird (Kagel/Roth 1995).

Die Bürokratietheorie wurde dagegen kaum weiterentwickelt. Es wurden einige weitere Modelle in der Art des Niskanen-Ansatzes vorgelegt, die sich vor allem darin unterscheiden, welchen Maximanden (Budget, Arbeit, Konfliktvermeidung) sie annehmen (z.B. Breton/Wintrobe 1975, Migué/Bélanger 1974). In der Folge basierten RC-Theori- 
en zum Verhalten in Bürokratien und Unternehmen jedoch stärker auf Prinicipal-agentModellen und Überlegungen aus dem Umfeld der Institutionenökonomie. Ein anders orientierter und in den Sozialwissenschaften besonders einflussreicher Beitrag zum Verhalten in Organisationen stammt von Hirschman (1970), der sich mit der Reaktion auf unbefriedigende Leistungen einer Organisation befasste. Er unterscheidet zwei grundlegende Reaktionsmöglichkeiten: Abwanderung (das am Markt übliche Verhalten) und Widerspruch (die Reaktion in politischen Organisationen).

Breit rezipiert wurde auch die an die Transaktionskostentheorie anknüpfende Theorie des institutionellen Wandels von North (1990). Er unterscheidet drei grundlegende Institutionen: Kultur, Recht und staatliche Gewalt, die jeweils in einer „Symbiose“ leben. Diese Symbiose dürfe jedoch nicht als statisch betrachtet werden. Nehmen einzelne Akteure die Chance auf Einkommenszuwächse wahr, werden sie versuchen, über Verhandlungen die Relationen zu verändern. Diese Prozesse werden getrieben durch das Bemühen, Transaktionskosten zu verringern, um im Wettbewerb mit anderen Staaten bestehen zu können.

Autokratien wurden in der PÖ lange vernachlässigt. Tullock präsentierte 1987 eine erste ökonomische Theorie der Autokratie, in welcher er die Autokratie aus der Perspektive des Diktators, der Macht und Einkommen maximiert, behandelt: Wie kommt er an die Macht, wie bleibt er an der Macht, und welche Politiken wählt er? Olson (1993) legte eine Theorie dazu vor, wie in einer Situation der Anarchie ein umherziehender Bandit zum Diktator werden kann, indem er zunächst die in kleinen Gruppen lebende Zivilbevölkerung ausraubt und sich dann zur Schutzmacht aufschwingt. Während Olson annimmt, dass der Diktator sein Einkommen maximiert, modelliert Wintrobe (1998) auch ideologische Ziele und Macht in der Nutzenfunktion. Nach beiden Theorien erreichen Diktatoren ihre Ziele dadurch, dass sie zum Wohlstand der Unterworfenen beitragen, was ihnen deren Loyalität sichert.

\subsubsection{Der Stand in Deutschland}

In Deutschland beschränkte sich die Beschäftigung mit den neuen politisch-ökonomischen Ansätzen zunächst auf die Rezeption und Aufarbeitung in Sammelbänden und Lehrbüchern. Außerdem wurden Hauptwerke der Theoretiker der NPÖ übersetzt. Lehner verfasste das erste deutsche politikwissenschaftliche Lehrbuch zur NPÖ (1981). ${ }^{1}$ In der deutschen Politikwissenschaft hat es seither nur noch von Braun (1999) eine lehrbuchartige Annäherung gegeben, die Darstellung und Diskussion der Theorien verbindet.

Eigene Anwendungen von NPÖ-Theorien für empirische Fragestellungen oder gar eine Weiterentwicklung der Modelle finden sich in den 1970er und 1980er Jahren in Deutschland kaum. Beispiele sind die Auseinandersetzung mit der Verfälschung bei demokratischen Entscheidungsregeln (Kern 1979), die Thematisierung politischer Kon-

1 Ein englischsprachiges Lehrbuch dieser Art (McLean) erschien immerhin erst 1987 und auch deutsche ökonomische Lehrbücher finden sich nicht wesentlich früher: Bernholz (1972, 1975, 1979; später Bernholz/Breyer (1993, 1994); Frey (1977, 1981) und Kirsch (1974; heute: 2004). 
junkturzyklen (Kirchgässner 1977; Rattinger/Puschner 1981), die Anwendung des rentseeking auf Probleme der Armut in der dritten Welt (Weede 1985) und die Anwendung der Bürokratietheorie auf die Umweltpolitik (Holzinger 1987).

Zur Koalitionstheorie und Wahlforschung finden sich aus der Perspektive der NPÖ zunächst vor allem die oben schon erwähnten Arbeiten von Pappi (1973; Kappelhoff/ Pappi 1983; Melbeck/Pappi 1984). In den 1990er und 2000er Jahren wuchs die politökonomische Literatur zur Wahl- und Parteienforschung sowie zum deutschen Regierungs- und Gesetzgebungssystem deutlich an. Im Feld der demokratischen Kollektiventscheidung entstanden theoretische Arbeiten zu Aggregationsmechanismen (List 2004), zu Abstimmungsregeln und ihren Problemen (Behnke/Zintl 2008) und zur Theorie des Parteienwettbewerbs (Behnke 1999; Bräuninger 2005; Martin/Plümper 2006). Daneben steht eine wachsende Zahl analytischer und empirischer Aufsätze zu Wahlen (Fuchs/ Kühnel 1994; Shikano 2006; Herrmann et al. 2006; Selb 2008), Wählern (Thurner/ Eymann 2000; Kroneberg 2006), Parteien (Manow 2003) und zum Parteienwettbewerb (Hainmüller et al. 2006). Häufig werden dabei Daten aus Deutschland verwendet. Viele Beiträge befassen sich mit deutschen Besonderheiten, etwa den Überhangmandaten (Behnke 2003) und den Folgen der Notwendigkeit der Koalitionsbildung im deutschen System (Pappi/Becker/Herzog 2005; Ganghof 2006; Linhart et al. 2008; Debus 2009; Linhart/Pappi 2009).

Schließlich sind die räumlichen Analysen zum deutschen Regierungssystem zu nennen. In der Tradition von Döring (1995; Döring/Hallerberg 2004) befassten sich seit den 1990er Jahren vor allem Wissenschaftler an der Universität Mannheim mit Vetospielern und Agendasetzern in Parteien, Parlamenten, Ausschüssen in Deutschland und der EU (z. B. Ganghof et al. 2009). Ein bedeutender Diskussionsgegenstand war die Wandlungsfähigkeit des deutschen Föderalismus. Während Scharpf (1985) das deutsche und europäische Mehrebenensystem in der Politikverflechtungsfalle sah und den Fokus der Analyse auf die Handlungsrestriktionen im deutschen Föderalismus legte, führten die Studien von Bräuninger und König zur Analyse des deutschen Zweikammersystems und die Messung des quantitativen Gesetzgebungsoutputs zu dem Ergebnis, dass der Reformstau weniger auf die föderalistische Struktur, sondern eher auf den Koalitionszwang und das Verhältniswahlrecht zurückzuführen sei (Bräuninger/König 1999; König/Bräuninger 1997; König 1997).

$\mathrm{Zu}$ anderen Gegenständen des Teilgebiets Politische Systeme, etwa zu Interessengruppen, Verwaltungen oder Gerichten wurden seit 1990 nur wenige RC-orientierte Arbeiten veröffentlicht. Ein Beispiel aus dem Bereich der Interessengruppen ist die Studie von Pappi, König und Knoke zum Interessengruppeneinfluss in der deutschen und amerikanischen Arbeits- und Sozialpolitik (1995), ein anderes die Arbeit von Henning (2000) zur europäischen Agrarpolitik, die das bäuerliche Lobbying im Sinne einer positiven Theorie von Kollektiventscheidungen analysiert. Andere Beispiele sind Hönnige (2007) zu Verfassungsgerichten oder das Jahrbuch Handlungs- und Entscheidungstheorie zu Verfassungsreformen (Shikano et al. 2009). 
3.3 Policy-Forschung und Politische Ökonomie

\subsubsection{Der internationale Stand}

Die Policy-Analyse ist generell kein großes Feld für die Anwendung des RC-Ansatzes. Grundsätzlich ist das Konzept des Policy-Zyklus zwar vereinbar mit RC-Annahmen. Policy-Studien können durchaus unter Einsatz des RC-Instrumentariums durchgeführt werden. Auch international überwiegen aber in der Policy-Analyse beschreibende Einzelfallstudien oder explanativ angelegte vergleichende Fallstudien. Ein methodischer Ansatz, bei dem versucht wurde, Policy-Studien und RC zu vereinen, ist das Konzept der analytical narratives von Bates et al. (1998). Die Idee ist hier, Fallstudien als analytische Rekonstruktionen mit Hilfe von RC-basierten Modellen durchzuführen, sodass mehrere Fallstudien vergleichbar und auf diese Weise Meta-Analysen möglich werden.

Dennoch gibt es eine große Zahl von Studien, die aus der Perspektive der NPÖ ein Politikfeld analysieren. Dies gilt besonders für das Feld der Wirtschaftspolitik. Die hauptsächlichen Organe für diese Studien sind das Journal of Political Economy und das European Journal of Political Economy. Hier publizieren zwar überwiegend Ökonomen, doch auch ein ansehnlicher Prozentsatz amerikanischer und europäischer Politikwissenschaftler. Das Gebiet ist zu breit, um hier sinnvoll einen Überblick geben zu können.

\subsubsection{Der Stand in Deutschland}

Auch in Deutschland gibt es Policy-Analysen, die aus der RC-Perspektive durchgeführt werden. Frühe Beispiele sind Lehner et al. zur Bankenpolitik (1983), Keck (1984) zur Technologiepolitik sowie Holzinger (1987) und Horbach (1992) zur Umweltpolitik. Scharpf (1988) legte eine Analyse von Inflation und Arbeitslosigkeit in Westeuropa vor, in der er das spieltheoretische Instrumentarium einsetzte.

Der akteurzentrierte Institutionalismus bildete den Hintergrund für eine größere Zahl von Policy-Analysen in verschiedenen Feldern, die im Kölner Max-Planck-Institut für Gesellschaftsforschung entstanden: Technologiepolitik (Schneider 1989, 1999; Schmidt/ Werle 1998), Gesundheitspolitik (Döhler 1990; Döhler/Manow 1997), internationale Steuerpolitik (Genschel 2002; Ganghof 2006) und internationale Finanzmärkte (Lütz 2002). Ähnliche Arbeiten entstanden unter der Leitung von Adrienne Héritier im späteren Max-Planck-Institut zur Erforschung von Kollektivgütern (z.B. in Héritier 2002). Meine eigene Studie zu transnationalen Gemeinschaftsgütern im Bereich der Finanzmärkte und der Umweltpolitik lässt sich als eine Sammlung von analytical narratives auffassen (Holzinger 2008). Schneider und Janning (2006) stellen neben den akteurzentrierten Institutionalismus und den RC-Institutionalismus den „,beziehungsstrukturellen Ansatz" als einen auf die soziologische RC-Theorie gestützten Ansatz der Policy-Analyse.

Ab den 2000er Jahren häufen sich Beiträge, die sowohl mit einer RC-Perspektive arbeiten als auch wirtschaftspolitische Gegenstände untersuchen, die also im Überschneidungsbereich der beiden eingangs unterschiedenen Formen von PÖ liegen. Bei- 
spiele umfassen Arbeiten zur internationalen Handelspolitik (Martin 2004), zu Staatsausgaben und Wirtschaftswachstum (Martin/Plümper 2003), zu öffentlichen Infrastrukturinvestitionen und wirtschaftlicher Performanz in deutschen Städten (Kemmerling/Stephan 2002) und zu Parteipolitik und Aktienkursen (Füss/Bechtel 2008). Diesen Studien ist gemeinsam, dass sie Zusammenhänge zwischen strategischem Handeln politischer Akteure und makro-ökonomischen Größen untersuchen und dazu analytische Modelle und quantitative Tests einsetzen.

\subsection{Komparatistik}

\subsubsection{Der internationale Stand}

Überraschenderweise fasste die NPÖ auch in der Komparatistik relativ spät Fuß (Mahoney 2000). Überraschend deshalb, weil die institutionellen Analysen der NPÖ, z.B. zu den Abstimmungsregeln und zum Parteienwettbewerb, eigentlich zum Vergleich geradezu einluden. In der Komparatistik überwogen jedoch nach dem zweiten Weltkrieg die typologischen Ansätze: etwa von Dahl (1971) und in jüngerer Zeit Lijphart (1992). Die Institutionensysteme wurden vergleichend beschrieben, und es wurden quantitative ländervergleichende Analysen zur Performanz verschiedener Systeme durchgeführt (Lijphart 1999).

Einige frühe Versuche, Koalitionsbildung vergleichend zu analysieren (de Swan 1973) blieben weitgehend unbemerkt. Erst in den 1990er Jahren wurden Koalitions- und Regierungsbildung bei den RC-Forschern zum Thema (Strom 1990; Laver/Shepsle 1996). Smith (1996) untersuchte vergleichend und mit den Mitteln der strategischen Spieltheorie die Wahl von Wahlzeitpunkten und Vertrauensvoten in parlamentarischen Systemen. Instabilität und innere Kriege wurden schon von Rabushka/Shepsle (1971) aufgegriffen, aber erst nach dem Beitrag von Fearon/Laitin (1996) zu interethnischer Kooperation wurde dieser Frage mehr Aufmerksamkeit geschenkt.

Aus einer NPÖ-Perspektive beschäftigen sich Persson und Tabellini (2003) mit den grundlegenden Institutionen wie Regierungs- und Wahlsystemen. Ihre Modelle sagen vorher, dass in Mehrheitssystemen weniger öffentliche Güter angeboten werden als in Verhältniswahlsystemen, dafür aber auch niedrigere Renten für die Politiker entstehen. Präsidiale Systeme beschränken das rent-seeking und sorgen für einen kleineren Staatssektor. Diese Hypothesen bestätigen sie mit Hilfe ländervergleichender Daten.

Erst im letzten Jahrzehnt hat ein Ansatz aus dem RC-Paradigma die Vergleichende Politikwissenschaft erobert: die Theorie der Vetospieler von George Tsebelis (2000). Tsebelis baut auf den zweidimensionalen räumlichen Politikmodellen auf und präsentiert gleichzeitig eine Weiterentwicklung der Koalitions- und der Verhandlungstheorie. Ein Vetospieler ist ein Akteur, dessen Zustimmung zur Veränderung des Status quo nötig ist. Die Einstimmigkeit als Grundelement der Verhandlungstheorie verbindet sich mit der räumlichen Darstellung, bei der die idealen Policy-Positionen der Akteure jeweils mit dem Status quo verglichen werden. Die relative Lage der Idealpositionen der Akteure zueinander und zum Status quo bestimmt des Ausmaß der möglichen Politikänderungen und damit die Politikstabilität. Die Politik ist (cum grano salis) umso stabiler, je mehr (institutionelle oder parteipolitische) Vetospieler ein politisches System auf- 
weist, je weiter entfernt deren Idealpositionen voneinander und je näher diese wiederum am Status quo liegen. Da sich politische Systeme nach der Zahl der Vetospieler klassifizieren lassen, hat diese Theorie rasch Eingang in die Komparatistik gefunden.

\subsubsection{Der Stand in Deutschland}

Die Entwicklung in Deutschland verlief ganz ähnlich. Auch hier dominierten zunächst institutionelle Klassifikationen (z. B. Hättich 1966; Steffani 1979). Auch in Deutschland wurde die Typologie von Lijphart zum Kernbestand der Komparatistik und auch hier wurden quantitative Performanz-Vergleiche von politischen Systemen durchgeführt (z. B. Schmidt 1992; Freitag/Vatter 2007). RC-Instrumente spielten keine Rolle beim Vergleich.

Die Theorie der Vetospieler wurde dann aber rasch in den Kanon der Vergleichenden Politikwissenschaft aufgenommen. Das Kernkonzept, die Rolle der Vetospieler als Blockierer von Reformen, wurde vor Tsebelis schon von einer in Deutschland arbeitenden amerikanischen Forscherin formuliert (Immergut 1990). Ganghof diskutiert die theoretischen und methodischen Probleme des Konzepts der Vetospieler (2003), wendet es aber wie andere Komparatisten auch in der eigenen, vergleichenden politisch-ökonomischen Forschung an (z. B. Ganghof et al. 2009; Zohlnhöfer 2001). In quantitativen ländervergleichenden Studien wird der Einsatz einer Vetospieler-Variable in den letzten Jahren zum Standard nicht nur in der politisch-ökonomischen Forschung, sondern immer auch dann, wenn es um Politikwandel (Zohlnhöfer 2009) und transnationale Politikkonvergenz geht (Holzinger et al. 2007).

Deutsche Forscher haben aber auch zur Weiterentwicklung der Theorie und ihrer empirischen Anwendbarkeit beigetragen. Die Vetospieler-Theorie basiert auf der relativen Lage der idealen Policy-Positionen der Akteure und auf den durch den Status quo verlaufenden Indifferenzkurven. Ein großes Problem für die Vorhersagekraft der Theorie ist die empirische Messung der Lage der Idealposition und des Status quo. Ein weiteres Problem liegt in der Annahme, die der Form der Indifferenzkurven zugrunde liegt, nämlich dass die Präferenzen der Akteure hinsichtlich der betrachteten Politikdimensionen gleich intensiv sind. Verfahren zur Messung von Policy-Positionen sind vor allem von König, Bräuninger und deren Ko-Autoren entwickelt worden (z. B. Bräuninger/König 2000; König 2005; Junge/König 2007; König/Finke 2007). König und Finke haben aber auch die Theorie weiterentwickelt, insofern sie elliptische Indifferenzkurven eingeführt und empirisch messbar gemacht haben (Finke 2009a, b).

Einige Arbeiten der vergleichenden PÖ aus einer RC-Perspektive wurden in dem Band von Obinger et al. (2003) systematisch zusammengestellt. Neben allgemeinen PÖ-Modellen zur Demokratie (Plümper) und zum Parteienwettbewerb (Zohlnhöfer) werden mit den Arbeitsbeziehungen (Kittel), der Arbeitslosigkeit (Armingeon), dem Wirtschaftswachstum (Obinger), der Inflation (Busch), der Währung (Freitag), der AuBenwirtschaft (Martin), den Steuern (Wagschal), der Verschuldung (Wagschal), den Staatsausgaben (Cusack/Fuchs) und dem Wohlfahrtsstaat (Obinger/Kittel) alle wesentlichen Bereiche der makroökonomischen Steuerung behandelt. Bei dieser Autorengruppe dominiert zwar der empirische Zugang zu ökonomischen Politiken, doch präsentieren fast alle Artikel auch politisch-ökonomische Modelle. 


\subsection{Internationale Beziehungen}

\subsubsection{Der internationale Stand}

Obwohl Morgenthau (1948) sich in einer ersten Version des Realismus dezidiert gegen die dem Idealismus der Zwischenkriegszeit innewohnende Idee einer Vernunft wandte, die Staaten zur Herstellung einer friedlichen internationalen Ordnung veranlassen könnte, sind spätere Theorien der Internationalen Beziehungen affin gegenüber dem RCAnsatz. Dies gilt schon für spätere Versionen des Realismus und für den Neorealimus (Waltz 1979). Auch wenn die methodologischen Fundamente des Realismus nicht sehr klar expliziert wurden, so wurden doch die Instrumente der politischen Ökonomie (Mikroökonomie und Spieltheorie) sehr bald eingesetzt. Beispiele sind die Entwicklung der Abschreckungstheorien, das Sicherheitsdilemma oder die Interpretation von Kriegen als Verhandlungsprozesse (z. B. Fearon 1995; Wagner 2007). Dies gilt in ähnlicher Weise für den Liberalismus. Beispiele sind die These von der Möglichkeit der Kooperation im wiederholten Gefangenendilemma (Axelrod 1984) oder die These vom demokratischen (Small/Singer 1976; Schultz 2001) oder freihändlerischen Frieden (Polachek 1980). Jenseits der theoretischen Auseinandersetzung zwischen Neorealisten und neoliberalen Institutionalisten finden sich auf beiden Seiten RC-Theoretiker. Auch die Ausdifferenzierung und Weiterentwicklung der Methoden wurde in den Internationalen Beziehungen mit vollzogen, wie das Lehrbuch von Bueno de Mesquita (2000) zeigt.

PÖ spielt auch als Gegenstandsbereich eine große Rolle in den Internationalen Beziehungen. Vor allem drei Gebiete sind es, zu denen Modelle und empirische Tests entwickelt werden: die klassische Außenhandels- und Wachstumstheorie (Rogowski 1987), die sich auf die ökonomische Außenwirtschaftstheorie stützt; die Entwicklungstheorie, in der von Modernisierung über Interdependenz bis zu neuen institutionalistischen Theorien ein bunter Strauß an Theorien diskutiert wird (Lipset 1959; Dos Santos 1969; Galtung 1971; Olson 2000); und die Globalisierungstheorie, in deren Rahmen die Liberalisierung des Handels, der Finanzmärkte, der Steuer- und Regulierungswettbewerb, die Rolle transnationaler Kollektivgüter und vor allem die Frage globaler Ungleichheit (Keohane/Milner 1996; Bretschger/Hettich 2002; Quinn 1997; Rodrik 1998; Sala-i-Martin 2006) behandelt werden.

International gesehen ist die Subdisziplin der Internationalen Beziehungen sicher der Bereich, in dem RC-Ansätze am stärksten verankert sind. Die Methoden-Grundsatzdiskussion wurde parallel auch in diesem Feld geführt (Kahler 1998). Mit dem Konstruktivismus entstand dem RC-Ansatz in den Internationalen Beziehungen erstmals ein einheitlicher Gegenspieler (Marx 2006). Vier Argumente werden gegen den ökonomischen Ansatz ins Feld geführt. (1) Präferenzen dürften nicht als modell-exogen behandelt werden, sie seien endogen und unterlägen dem Wandel; (2) Was Individuen wahrnehmen, was sie wollen und wie sie handeln, sei durch die Kultur bedingt; (3) Die Identität von Akteuren sei sozial konstruiert und deshalb gegenüber Präferenzen und Handlungen vorrangig (z.B. Jeppersen/Katzenstein/Wendt 1996); (4) Verhalten sei nicht durch Eigeninteresse, sondern durch soziale Normen und die Orientierung an angemessenem Handeln geleitet (z. B. Checkel/Moravcsik 2001). Die weitere Diskussion deutet 
an, dass bestimmte Kritiken nicht grundsätzlich mit dem RC-Ansatz inkompatibel sind (Greif 1995; Kahler 1998; Fearon/Wendt 2002).

\subsubsection{Der Stand in Deutschland}

Zwar gab es in Deutschland im Teilbereich der Internationalen Beziehungen keineswegs nur Idealisten und Konstruktivisten, sondern durchaus viele Vertreter des Realismus und Liberalismus. Dennoch gibt es bis heute nur wenige, die das Instrumentarium der PÖ anwenden, formale Theoriebildung betreiben oder quantitative empirische Tests zu politisch-ökonomischen Fragestellungen durchführen. Außer zwei Aufsätzen amerikanischer Autoren (Singer 1969; Rapoport 1970) finden sich in der PVS bis 1980 keine Hinweise auf ökonomische Ansätze zur internationalen Politik. Der Soziologe Weede beschäftigte sich 1985 mit rent-seeking und Armut in den Entwicklungsländern, und 1991 erschien ein Aufsatz von Keck zum neuen Institutionalismus in der Theorie der Internationalen Politik. Auch in diesem Jahrzehnt gibt es keine größere Zahl von Beiträgen zu internationalen Themen in der PVS, deren Autoren mit dem RC-Ansatz arbeiten oder Internationale PÖ betreiben (Ausnahmen: Plümper 2001; Bussmann et al. 2003; Martin 2004; Sattler/Walter 2008).

Die geringe Präsenz in der PVS könnte der Tatsache geschuldet sein, dass Autoren aus diesem Bereich in der Zeitschrift für Internationale Beziehungen (ZIB) oder in englischsprachigen Zeitschriften publizieren. Tatsächlich argumentieren in der ZIB rund zehn Prozent der Beiträge aus einer RC-Perspektive, und rund 25 Prozent der Beiträge widmen sich Themen der Internationalen PÖ. Hinsichtlich des RC-Paradigmas erscheint das immer noch wenig, wenn man in Betracht zieht, dass die meisten dieser Aufsätze im Rahmen der sogenannten ZIB-Debatte erschienen - einer Debatte, die mit dem ersten Heft der ZIB 1994 begann und bis heute andauert.

Die ZIB-Debatte wird als eine Diskussion zwischen Rationalismus und Konstruktivismus geführt (Schäfer 2007; Grobe 2007). Sie ist eine Theoriedebatte, ein unmittelbarer Bezug zu Gegenständen der Internationalen Beziehungen taucht eher selten auf. Die Diskussion weicht insofern von der internationalen Debatte ab, als das Konzept des verständigungsorientierten Handelns von Habermas im Mittelpunkt steht. Gleichzeitig werden das norm-orientierte Individuum der Akteursfiktion des RC gegenübergestellt und einige klassische Kritikpunkte am RC-Zugang verhandelt. Die Grundfrage des philosophischen Konstruktivismus, der Umgang mit (sozial) konstruierter Wahrnehmung, wird dagegen kaum berührt. Es geht um die Rolle von Kommunikation (Müller 1994): Ist sie nur ,cheap talk“ oder bindet sie (Risse-Kappen 1995; Schmalz-Bruns 1995)? Welche Rolle kann sie in RC-Analysen spielen (Schneider 1994; Keck 1995; Müller 1995)? Wie verhalten sich Argumentieren und Verhandeln, verständigungsorientierte und strategische Kommunikation (von Prittwitz 1996; Zangl/Zürn 1996; Holzinger 2001)? Gibt es mit dem „rhetorischen Handeln“ (Schimmelfennig 1997) nicht doch etwas dazwischen? Was ist die Rolle moralischer Argumente (Hanrieder 2008)?

Einen eigenen Beitrag zum Forschungsinstrumentarium hat Zürn mit seinem ,situationsstrukturellen Ansatz" geleistet (1992). Der Ansatz nimmt die RC-Annahmen und -Techniken in moderater Form auf und ähnelt darin dem akteurzentrierten Institutionalismus. Er betont allerdings weniger die Institutionen als die situativ gegebene Ak- 
teurskonstellation. Der akteurzentrierte Institutionalismus, der situationsstrukturelle Ansatz und die Studien von Ostrom $(1990,2005)$ zur Lösung von lokalen Kollektivgutproblemen haben gemeinsam, dass sie die hoch abstrakten Situationsanalysen der RCKlassiker erweitern, indem sie Annahmen über die konkrete Situation einfließen lassen.

\subsection{Europäische Integration und EU}

\subsubsection{Der internationale Stand}

Mit der Europäischen Union befassen sich mehrere Teildisziplinen. Innerhalb der Internationalen Beziehungen wird die EU vor allem unter dem Aspekt der regionalen Integration behandelt. Der eigentliche Fortschritt in der Analyse der EU erfolgte jedoch im Bereich der Systemforschung. Hier entwickelte sich seit Beginn der 1990er Jahre ein Programm des RC-Institutionalismus, das dem der amerikanischen RC-Analysen nicht nachsteht. An dieser Forschung sind amerikanische und europäische Autoren beteiligt.

Die Studien beziehen sich sowohl auf das Verhältnis der europäischen Organe untereinander als auch auf die einzelnen Organe: Ministerrat, Kommission, Europäisches Parlament, Gerichtshof. Zwei Instrumentarien werden vorwiegend eingesetzt: Machtindizes und räumliche Modelle. Strategische Spiele in extensiver Form, Variation der Informationsannahmen oder wiederholte Spiele finden sich seltener als Analysemittel.

Anwendungen von Machtindizes (Maße für die Abstimmungsmacht in Gremien mit Stimmgewichtung) auf den europäischen Ministerrat gab es schon früh (Weiersmüller 1971; Brams/Affuso 1976). Die Machtindizes erlebten seit Beginn der 1990er Jahre einen Aufschwung (z. B. Holler 1981; Nurmi 1987; Hosli 1996; Lane/Maeland 1995; Widgren 1994), da ihre Berechnung inzwischen regelmäßig als Dienstleistung von Regierungen nachgefragt wird. Auch auf das Parlament (Stärke der Fraktionen und der nationalen Gruppen) wurden Machtindizes angewendet (Herne/Nurmi 1993; Hosli 1997). Relativ erfolglos blieben Versuche, interinstitutionelle Machtindizes zu ermitteln (Herne/Nurmi 1993; Nurmi/Maskanen 1999; vgl. Dowding 2000).

In einer von Garrett/Tsebelis (1996) ausgelösten Kontroverse wurden den Machtindizes räumliche Modelle gegenübergestellt. Räumliche Modelle können einerseits den Nachteil der Machtindizes ausgleichen, bei der Koalitionsanalyse blind für die Ähnlichkeit von Präferenzen zu sein, andererseits sind sie auch in der Lage, interinstitutionelle Machtanalysen zu leisten. Dies erkaufen sie allerdings mit groben Vereinfachungen der Entscheidungsverfahren und mit nicht immer überzeugend zu rechtfertigenden Annahmen über die relativen Präferenzen der Akteure (z. B. Tsebelis 1994, 1995, 1996, 2000; Tsebelis/Garrett 1997; Steunenberg 1994; Crombez 1997). Die Modelle repräsentieren die wichtigsten Entscheidungsverfahren der EU (Konsultation, Kooperation, Kodezision) und kommen zu unterschiedlichen Policy-Vorhersagen und Ergebnissen über die relative Macht der Akteure. Empirische Tests mit großen Datensätzen wurden bisher nur selten durchgeführt (Pajala/Widgrén 2004).

Zur internen Analyse von Parlament und Kommission liegen weniger Arbeiten vor. Hix untersuchte die Fraktionen und Parteien im Parlament und ihr Stimmverhalten in den Dimensionen rechts-links und pro- und anti-europäische Integration (z. B. 1996, 
1998). Sein Hauptergebnis ist, dass die europäischen Parteizusammenschlüsse sich zunehmend wie Parteien verhalten und dass die nationale Orientierung der Abgeordneten zurückgeht. Außerdem gibt es einige Koalitionsanalysen (Laver 1997; Laver/Shepsle 1996). Im Vergleich zu den Analysen des amerikanischen Kongresses sind RC-Analysen des Europäischen Parlaments aber noch wenig ausdifferenziert. Ähnliches lässt sich für interne Untersuchungen der Kommission sagen: Hier gibt es einige wenige Analysen aus einer Principal-agent-Perspektive (Dunleavy 1997; McCubbins/Page 1987) und Modelle des Komitologie-Prozesses (Franchino 2000). Auch der Europäische Gerichtshof hat die Aufmerksamkeit der RC-Forscher nur in geringem Maß auf sich gezogen (z.B. Garrett 1992; Garrett/Weingast 1993; Burley/Mattli 1993; Slaughter 1993), ebenso wie die europäischen Interessengruppen und das rent-seeking (Schneider/Baltz 2003).

\subsubsection{Der Stand in Deutschland}

An der institutionellen Analyse der EU-Entscheidungsverfahren beteiligten sich einige deutsche Forscher schon ab Beginn der 1990er Jahre, also gleichzeitig mit der internationalen Welle (z. B. Holzinger 1994; Schneider 1995; Schneider/Seybold 1997). Ein von Moser, Schneider und Kirchgässner (1997) herausgegebenes Sonderheft der Zeitschrift Aussenwirtschaft fasst eine Reihe von Beiträgen meist europäischer Autoren unter dem Titel „Decision Rules in the European Union: A Rational Choice Perspective“ zusammen. In diesen Artikeln werden das Zusammenwirken der europäischen Institutionen und die Verteilung von Macht analysiert, aber auch die interne Funktionsweise der einzelnen Organe.

Ab Ende der 1990er Jahre sind es dann besonders die Gruppen um König und Bräuninger sowie Schneider und Ko-Autoren (Schneider/Bailer 2000, 2002; Schneider/Baltz 2003), die sich an der RC-basierten Analyse der europäischen Entscheidungsprozesse beteiligen. Gegenstände der Analyse sind die Entscheidungsregeln (König/Bräuninger 2000; König/Proksch 2006), die legislativen Verfahren (König 1996, 2008; Schulz/ König 2000), die Messung von Policy-Positionen (König 2005), Beiträge zur Vetospieler-Analyse der EU (König/Pöter 2001; König/Tröger 2005), die Osterweiterung (König/Bräuninger 2004) und die schon erwähnte Weiterentwicklung der räumlichen Modelle (König/Finke 2007; Junge/König 2007). Erwähnenswert ist ein jüngeres internationales Projekt „Domestic Structures and European Integration“, in dem alle Stufen des Konventsprozesses (Konvent, Vorabstimmung und Verhandlungen der Regierungskonferenz, Ratifikationsprozesse und Referenda) untersucht werden (König/Slapin 2006; Hug/König 2007; König et al. 2008). Die Konstitutionalisierung der EU und die Präferenz- und Kompromissbildung bei den Regierungskonferenzen ist auch Gegenstand von zwei Büchern (Thurner 2006; Thurner/Pappi 2009). Weitere institutionelle Analysen aus der RC-Perspektive befassen sich mit den Machtverhältnissen zwischen den Mitgliedstaaten (Beisbart et al. 2005) oder zwischen dem Europäischen Parlament und dem Rat (Kasack 2004).

Ein beträchtlicher Teil der eben genannten Aufsätze ist in der Zeitschrift European Union Politics erscheinen. Die Zeitschrift wurde von Gerald Schneider (Universität Konstanz) gegründet und bildet seither das wesentliche Forum für Analysen der euro- 
päischen Politik, die dem RC-Institutionalismus zuzurechnen sind. Dies gilt nicht nur für die deutsche, sondern auch für die internationale Diskussion zu diesen Themen. Primär publizieren angelsächsische Autoren, Schweizer und Skandinavier in der EUP. Der deutsche Anteil macht aber immerhin 15 bis 20 Prozent aus. Die Zeitschrift ist insofern sehr erfolgreich, als sie in den letzten Jahren regelmäßig unter den zehn weltweit besten politikwissenschaftlichen Zeitschriften geführt wird. ${ }^{2}$ Die EUP ist damit als ein eigener deutscher Beitrag zur internationalen EU-Forschung aus einer Perspektive der ökonomischen Theorien der Politik zu bewerten.

\section{Institutionen und Publikationen}

Ein abschließender Blick auf die institutionelle Verankerung der deutschen Forscher im Bereich der PÖ und auf ihre wichtigsten Publikationsorgane ermöglicht die Einschätzung ihrer Rolle innerhalb der deutschen Politikwissenschaft.

\subsection{Institutionelle Verankerung}

International haben sich die Vertreter der NPÖ vor allem in der Public Choice Society (seit 1964) organisiert, die auch eine europäische Unterorganisation hat. Ihr gehören durchaus auch deutsche politische Ökonomen an, sie ist jedoch dominiert durch Vertreter der Disziplin Ökonomie. Eine eigene Organisation der Politikwissenschaftler in diesem Feld gibt es nicht.

In der Deutschen Vereinigung für Politische Wissenschaft (DVPW) gibt es zum jetzigen Zeitpunkt mehrere Untergliederungen, in deren Rahmen PÖ verschiedener Provenienz betrieben wird. Die Sektion „Politik und Ökonomie“ führt ein bis zwei Tagungen im Jahr durch. Die Themen sind wirtschaftspolitischer Natur und variieren über die Zeit: Während in den 1980er Jahren z.B. Fragen der Arbeitslosigkeit und der öffentlichen Regulierung behandelt wurden, sind in den 1990er und 2000er Jahren der Wohlfahrtsstaat, Transformationsprozesse (Lütz/Czada 2004), die Schaffung europäischer und globaler Märkte (Czada/Lütz 2000) sowie die Entwicklung des Kapitalismus die beherrschenden Themen. Beiträge aus der Perspektive der NPÖ tauchen auf, sind aber nicht die Regel. Im Jahr 2003 hat sich die Ad-hoc-Gruppe Internationale Politische Ökonomie gegründet. Ihre Themen beschäftigen sich mit Globalisierungs-, Entwicklungs- und Umweltfragen. PÖ-Ansätze im theoretisch-methodischen Sinn kommen hier auch zum Tragen, es dominieren aber andere Ansätze.

Die NPÖ und der RC-Ansatz hatten ihre erste institutionelle Heimat im Arbeitskreis „Handlungs- und Entscheidungstheorie“, der 1993 gegründet wurde und der Sektion „Politische Theorie“ organisatorisch zugeordnet war. Auch dieser Arbeitskreis führt jährliche Tagungen durch und veröffentlichte die Vorträge zunächst regelmäßig in Sammelbänden (Druwe/Kunz 1994, 1996, 1998, 1999). Seit 2001 erscheint das „Jahrbuch für Handlungs- und Entscheidungstheorie“ (bisher vier Bände), in das Tagungsbeiträge und andere Manuskripte nach anonymer Begutachtung aufgenommen werden. Nach

2 Gemessen am sogenannten impact factor, z. B. von sciencewatch.com. 
inhaltlichen Schwerpunkten und Tagungsthemen lassen sich zwei Phasen des Arbeitskreises unterscheiden: Von 1993 bis 2000 war vor allem der RC-Ansatz selbst Gegenstand der Diskussion. Es dominierte die wissenschaftstheoretische Reflexion über die Theorien rationalen Handelns. Ab 2000 wandte sich der Arbeitkreis allmählich von der Metadiskussion ab und es mehrten sich Arbeiten, in denen politikwissenschaftliche Gegenstände mit Hilfe des RC-Ansatzes „erklärt“ wurden. Der AK hat sich inzwischen auch personell und organisatorisch von der Theorie-Sektion entfernt.

\subsection{Publikationsorgane}

Wo werden Arbeiten aus der PÖ in Deutschland publiziert? Hier ist zunächst einmal der Verlag Mohr in Tübingen zu nennen, der in seiner Reihe „Einheit der Gesellschaftswissenschaften“ die wichtigsten Bücher von amerikanischen Autoren übersetzt, ${ }^{3}$ aber auch eigene Werke deutscher Autoren in diesem Feld veröffentlicht hat. ${ }^{4}$ Bei Mohr erscheint seit einigen Jahren auch eine Reihe von Konferenz-Bänden, die sich jeweils einem RCDenker widmen, etwa Buchanan, Olson, Williamson, Hirschman, Schelling oder Elster (hrsg. von Pies und Leschke). Schließlich verlegt Mohr das Jahrbuch für NPÖ, das seit einigen Jahren als Conferences on New Political Economy geführt wird und bisher 25 Bände umfasst. Die Bände widmen sich jeweils einem Thema, etwa „International Conflict Resolution“, „European Governance“, „Power and Fairness“. Die Beiträge stammen überwiegend von Ökonomen.

Bei Leske + Budrich und im VS-Verlag erschienen die Bände bzw. das Jahrbuch des Arbeitskreises „Handlungs- und Entscheidungstheorie“. Außerdem gibt der VS-Verlag eine Buchreihe „Neue Politische Ökonomie“ heraus (z. B. Martin 2005; Hönnige 2007; Bechtel 2009). Weitere Reihen, in denen Monographien aus der PÖ erscheinen, sind „Scientia Nova“ (z. B. Green/Shapiro 1999; Axelrod 2000) im Oldenbourg Verlag, sowie die „Mannheimer Beiträge zur politischen Soziologie und positiven politischen Theorie" im Campus Verlag (z. B. Henning 2000; Bräuniger 2000; Kaiser 2002).

Welche Zeitschriften stehen Beiträgen aus der PÖ offen? Zwei Zeitschriften, in denen politisch-ökonomische Ansätze besonders stark vertreten sind, wurden schon erwähnt: Analyse \& Kritik als ein Organ der sozialwissenschaftlichen RC-Theorie und European Union Politics als ein Organ für die Analyse der Institutionen und politischen Prozesse in der EU. Auch die ZIB-Debatte wurde schon geschildert. Jenseits dieser Debatte erscheinen in der ZIB jedoch keineswegs besonders häufig Artikel, die dem RC-Paradigma zuzuordnen wären.

Ein Blick auf die bedeutendsten allgemeinen politikwissenschaftlichen Zeitschriften in Deutschland (Politische Vierteljahresschrift, Leviathan, Zeitschrift für Politikwissenschaft) ergibt ein ähnliches Bild. In der PVS erschienen von 1960 bis 1979 genau zwei Beiträge (von insgesamt rund 550), die sich mit Fragen der NPÖ befassen: Kirchgässner 1977 und Kern 1979. In den folgenden beiden Jahrzehnten findet man jeweils etwa acht

3 So z.B. Downs (1968); Olson (1968, 2002); Becker (1982); Buchanan (1984); North (1988, 1992); Williamson (1990); Brennan/Buchanan (1993); Ostrom (1999).

4 So z.B. Behrens (1986); Homann (1988); Kirchgässner (1991); Pies (1993); Ockenfels (1999). 
bis zehn Aufsätze (das sind rund fünf Prozent), die sich im engeren Sinn mit RC-Fragestellungen beschäftigen oder den Ansatz reflektieren. ${ }^{5}$ Ab 2001 erscheinen deutlich mehr Beiträge aus dieser Perspektive, insgesamt etwa 20 in acht Jahren, ${ }^{6}$ womit die Zehn-Prozent-Marke wohl überschritten ist. ${ }^{7}$

Im Leviathan finden sich zwar von Beginn an viele Aufsätze, die zum weiteren Gebiet der PÖ gehören, aber keine typischen Gegenstände oder Autoren der NPÖ. Ab der zweiten Hälfte der 1980er Jahre gibt es zwar Beiträge von Autoren, die dem RC-Ansatz nahestehen; ${ }^{8}$ sie sind dann aber anderen Gegenständen gewidmet. Ganz ähnlich ist die Lage in der Zeitschrift für Politikwissenschaft (seit 1996): Man findet durchaus viele Beiträge von Autoren, die dem RC zuzuordnen sind, aber gerade nicht mit typischen Themen. ${ }^{9}$ Immerhin gab es aber drei Aufsätze, die sich der Reflexion über den RCAnsatz widmen (Zangl/Zürn 1999; Busch 2000; Dylla 2007).

Damit bleibt der Anteil der Beiträge aus dieser Forschungsrichtung recht gering. Doch unterscheidet sich die deutsche Publikationslandschaft nicht fundamental von der angelsächsischen. Zwar wurde immer wieder behauptet, der RC-Ansatz nehme in der American Political Science Review (APSR) 25 Prozent oder gar 50 Prozent ein; dies scheint aber einer Zählung nicht unbedingt standzuhalten. Marsh/Savigny (2004) zählen für zwei Fünfjahres-Zeiträume (1975-1979; 1997-2002) die Beiträge in American Journal of Political Science, American Political Science Review, British Journal of Political Science und Political Studies. In der Reihenfolge der Zeitschriften finden sie für den ersten Zeitraum 12.5, 15, zwölf und 12.5 Prozent und für den zweiten Zeitraum 15, 21, neun und sechs Prozent dem RC-Ansatz zugehörige Beiträge. Es gibt also eine leichte Zunahme in den amerikanischen Zeitschriften, aber wir bewegen uns in einer Größenordnung zwischen zehn und 20 Prozent (Green/Shapiro 1994 finden 20-30 Prozent). Allerdings scheint die RC-Literatur durchaus rezipiert zu werden: Von den 35 meistzitierten Autoren sind 37 Prozent und von den 14 meistzitierten Büchern 33 Prozent diesem Feld zuzurechnen.

Hauptorgan der NPÖ ist seit 1966 die Zeitschrift Public Choice. Sie erscheint inzwischen in acht Heften pro Jahr im Unfang von ungefähr 1500 Seiten. Die Autoren sind überwiegend Ökonomen, aber auch Politikwissenschaftler, wobei die Amerikaner dominieren. Deutsche Politikwissenschaftler suchte man lange Zeit vergebens. Seit 2000 finden sich dann immerhin zehn Beiträge deutscher Politikwissenschaftler, ausnahmslos

5 Z.B. Rattinger (1981); Weede (1985); Keller (1988); Keck (1991); König (1992); Jordana (1992); Vatter/Nabholz (1995); Mensch (1996); König/Bräuniger (1999).

6 Z.B. Braun (2001); Pappi/Shikano (2001); Thurner/Stoiber (2002); Thurner et al. (2005); Manow (2002); Behnke (2003, 2005, 2008); Bussmann et al. (2003); Debus (2007); Sattler/ Walter (2008).

7 Ich habe die Abhandlungen und Forum-Beiträge durchgesehen; da die Zuordnung nicht immer ganz einfach ist, handelt es sich nur um grobe Angaben.

8 Z.B. Hirschman (1992, 1994, 1995); Zürn (2003); Genschel (2008); Genschel/Schlichte (1997); Genschel/Zangl (2008); Czada (1998); Manow (2004, 2006, 2007); Schimmelfennig (2004); Höpner (2007).

9 Z.B. Zimmerling (1996); Zohlnhöfer (2001, 2009); Holzinger/Knill (2001); Behnke (2003); Ganghof (2005); Wagschal et al. (2008). 
der jüngeren Generation. ${ }^{10}$ Dies bestätigt den Trend, der sich auch in der PVS zeigt: Der Ansatz der NPÖ in seiner modernen Form, und das heißt Anwendung des Ansatzes auf spezifische Fragestellungen und hohes Maß der Formalisierung, ist nun auch in der deutschen Politikwissenschaft zu einer gewissen Normalität geworden. Es handelt sich zwar nach wie vor um eine kleine Gruppe, aber die jüngere Generation spielt nunmehr auch international aktiv mit.

\section{Zusammenfassung und Ausblick}

Die klassischen Werke der NPÖ entstanden in den 1950er und 1960er Jahren (Mitchell 1999). Seither konzentriert sich die Forschung auf die Ausdifferenzierung und Anwendung der grundlegenden Modelle. Wenngleich die Theorien von Wirtschaftswissenschaftlern entwickelt wurden, beeinflussten sie zunächst weniger die Ökonomie als die US-amerikanische Politikwissenschaft. Dort entwickelte sich die positive politische Theorie zu einem bedeutenden Paradigma. Als ein Zeichen der zunehmenden Anerkennung von Public choice in der Wirtschaftswissenschaft darf die häufige Verleihung von Nobelpreisen an Vertreter dieser Richtung gewertet werden: Arrow (1972), Simon (1978), Buchanan (1986), Coase (1991), Becker (1992), North (1993), Kahnemann (2002), Aumann/Schelling (2005).

In Deutschland wurde die NPÖ sehr zögerlich aufgenommen. In der Politikwissenschaft fasste anfangs lediglich das spieltheoretische Instrumentarium in den Internationalen Beziehungen Fuß. Inzwischen mehren sich jedoch auch in der deutschen Politikwissenschaft die Anzeichen für ein gesteigertes Interesse am RC-Ansatz. In jüngster Zeit finden sich viele Beiträge zur Wahl- und Parteienforschung, zur Analyse von Entscheidungsverfahren in der EU und zur Theorie der Veto-Spieler und ihrer empirischen Anwendung in Deutschland und Europa.

Die deutsche politisch-ökonomische Forschung war lange Zeit eher rezeptiv und anwendend. Bedeutsame Außenwirkung hatten lediglich das Konzept des akteurzentrierten Institutionalismus, das besonders in Europa rezipiert wurde, die international sichtbaren Beiträge zur Forschung zu politischen Systemen, Internationalen Beziehungen und zur EU aus Mannheim und Konstanz und die Gründung der Zeitschrift European Union Politics, in der mehr Amerikaner als Europäer publizieren.

In den meisten Teildisziplinen bilden die RC-Forscher zwar nach wie vor eine Minderheit. In einem wachsenden Kreis haben sich die typischen Werkzeuge aber zum Standardinstrumentarium entwickelt. In weicherer Form werden die Grundkonzepte zunehmend auch von Forschern benutzt, die nicht in einem engeren Sinn zur RC-Gemeinde gehören. Mit Ausnahme der politischen Theorie lässt sich eine größere Offenheit gegenüber dem Ansatz und weniger offene Ablehnung als noch zu Beginn der 1990er Jahre feststellen. Auch die Selbstzuordnung zum RC-Paradigma ist inzwischen geradezu überraschend hoch: In der jüngsten DVPW-Erhebung zum Stand der Disziplin im

10 Thurner/Eymann (2000); Kemmerling/Stephan (2002); Plümper/Martin (2003); List (2004); Bräuninger (2005); Schneider (2007); Strulik (2007); Füss/Bechtel (2008); Selb (2008); Debus (2009). 
Jahr 2006 gaben 41 Prozent der Befragten an, dass sie ihre Forschungen stark oder sehr stark auf den RC-Ansatz stützen. Nur der Neo-Institutionalismus und der historische Ansatz lagen mit 64 bzw. 45 Prozent noch höher. Zudem nannten 31 Prozent die Entscheidungstheorie und 20 Prozent die Spieltheorie (Falter/Knodt 2007). Dies lässt für die Zukunft mehr Forschung zur PÖ in Deutschland und mehr Beiträge zum internationalen Stand der Forschung in diesem Gebiet erwarten.

\section{Literatur}

Alt, James E. und Kenneth A. Shepsle (Hrsg.). 1990. Perspectives on Positive Political Economy. New York: Cambridge University Press.

Amadae, Sonja M. und Bruce Bueno de Mesquita. 1999. The Rochester School: The Origins of Positive Political Theory. Annual Review of Political Science 2: 269-295.

Arrow, Kenneth J. 1951. Social Science and Individual Values. New York: Wiley \& Sons.

Axelrod, Robert. 1984. The Evolution of Cooperation. New York: Basic Books.

Bates, Robert H., Avner Greif, Margaret Levi, Jean-Laurent Rosenthal und Barry R. Weingast. 1998. Analytic Narratives. Princeton: Princeton University Press.

Bechtel, Michael M. 2009. Regierung, Rendite, Risiko. Die politische Ökonomie des Aktienmarktes in Deutschland. Wiesbaden: VS Verlag für Sozialwissenschaften.

Becker, Gary S. 1976. The Economic Approach to Human Behavior. Chicago: Chicago University Press.

Behnke, Joachim. 1999. Räumliche Modelle der sachfragenorientierten Wahlentscheidung. Hamburg: Dr. Kovac.

Behnke, Joachim. 2003. Ein integrales Modell der Ursachen von Überhangmandaten. Politische Vierteljahresschrift 44: 41-65.

Behnke, Joachim. 2005. Lassen sich Signifikanztests auf Vollerhebungen anwenden? Einige essayistische Anmerkungen. Politische Vierteljahresschrift 46: O1-O15.

Behnke, Joachim. 2008. Strategisches Verhalten bei der Nachwahl in Dresden zur Bundestagswahl 2005. Politische Vierteljahresschrift 49: 695-720.

Behnke, Joachim und Reinhard Zintl. 2008. Condorcet. Ausgewählte Schriften zu Wahl- und Entscheidungsverfahren. Tübingen: Mohr.

Behrens, Peter. 1986. Die ökonomischen Grundlagen des Rechts. Tübingen: Mohr.

Beisbart, Claus, Luc Bovens und Stephan Hartmann. 2005. A Utilitarian Assessment of Alternative Decision Rules in the Council of Ministers. European Union Politics 6: 395-418.

Benz, Arthur, Fritz W. Scharpf und Reinhard Zintl. 1992. Horizontale Politikverflechtung. Zur Theorie von Verhandlungssystemen. Frankfurt a. M.: Campus.

Bernholz, Peter. 1972, 1975, 1979. Grundlagen der politischen Ökonomie. Band 1, 2 und 3. Tübingen: Mohr Siebeck.

Bernholz, Peter und Friedrich Breyer. 1993. Grundlagen der Politischen Ökonomie. Band 1: Theorie der Wirtschaftssysteme. Tübingen: Mohr Siebeck.

Bernholz, Peter und Friedrich Breyer. 1994. Grundlagen der Politischen Ökonomie. Band 2: Ökonomische Theorie der Politik. Tübingen: Mohr Siebeck.

Black, Duncan. 1958. The Theory of Committees and Elections. London/New York: Cambridge University Press.

Brams, Steven J. und Paul J. Affuso. 1976. Power and Size: A New Paradox. Theory and Decision 7: 29-56.

Braun, Dietmar. 1999. Theorien rationalen Handelns in der Politikwissenschaft. Opladen: Leske + Budrich. 
Braun, Dietmar. 2001. Intergouvernementale Beziehungen und Fiskalpolitik in Bundesstaaten. Politische Vierteljahresschrift 42: 624-654.

Bräuninger, Thomas. 2000. Internationale Institutionenpolitik. Die Wahl von Entscheidungsregeln für die Meeresbodenbehörde. Frankfurt a. M.: Campus.

Bräuninger, Thomas. 2005. A Partisan Model of Government Expenditure. Public Choice 125: 409-429.

Bräuninger, Thomas und Thomas König. 1999. The Checks and Balances of Party Federalism. German Federal Government in a Divided Legislature. European Journal of Political Research 36: 207-234.

Bräuninger, Thomas und Thomas König. 2000. Making Rules for Governing Global Commons. The Case of Deep-Sea Mining. Journal of Conflict Resolution 44: 604-629.

Brennan, Geoffrey und James M. Buchanan. 1980. The Power to Tax. Cambridge: Cambridge University Press.

Brennan, Geoffrey und James M. Buchanan. 1985. The Reason of Rules. Constitutional Political Economy. Cambridge: Cambridge University Press.

Brennan, Geoffrey und Alan Hamlin. 2000. Democratic Devices and Desires. Cambridge: Cambridge University Press.

Brennan, Geoffrey und Loren Lomaski. 1993. Democracy and Decision. The Pure Theory of Electoral Preference. Cambridge/New York: Cambridge University Press.

Breton, Albert und Ronald Wintrobe. 1975. The Equilibrium Size of a Budget-maximizing Bureau: A Note on Niskanen's Theory of Bureaucracy. Journal of Political Economy 83: 195207.

Bretschger, Lucas und Frank Hettich. 2002. Globalisation, Capital Mobility and Tax Competition: Theory and Evidence for OECD-Countries. European Journal of Political Economy 18: 695716

Buchanan, James M. 1975. The Limits of Liberty: Between Anarchy and Leviathan. Chicago/ London: University of Chicago Press.

Buchanan, James M. 1984. The Ethical Limits of Taxation. Scandinavian Journal of Economics 86: $102-14$

Buchanan, James M. und Gordon Tullock. 1962. The Calculus of Consent. Logical Foundations of Constitutional Democracy. Ann Arbor: University of Michigan Press.

Bueno de Mesquita, Bruce. 2000. Principles of International Politics: People's Power, Preferences, and Perceptions. Washington D.C.: Congressional Quarterly Press.

Burley, Anne-Marie und Walter Mattli. 1993. Europe Before the Court: A Political Theory of Legal Integration. International Organization 47: 41-76.

Busch, Andreas. 2000. Durch Wandel zur Annährung? Politische Ideen und Rational Choice. Zeitschrift für Politikwissenschaft 10: 999-1014.

Bussman, Margit, Harald Scheuthle und Gerald Schneider. 2003. Die „Friedensdividende“ der Globalisierung: Außenwirtschaftliche Öffnung und innenpolitische Stabilität in den Entwicklungsländern. Politische Vierteljahresschrift 44: 302-324.

Checkel, Jeffrey T. und Andrew Moravcsik. 2001. A Constructivist Research Program in EU Studies? European Union Politics 2: 219-249.

Coase, Ronald H. 1960. The Problem of Social Cost. Journal of Law and Economics 3: 1-44.

Coleman, James S. 1994. Foundations of Social Theory. Cambridge: Harvard-Belknap Press.

Crombez, Christophe. 1997. Policy Making and Commission Appointment in the European Union. Aussenwirtschaft 52: 63-82.

Czada, Roland. 1998. Vereinigungskrise und Standortdebatte. Leviathan 26: 24-59.

Czada, Roland und Susanne Lütz (Hrsg.). 2000. Die politische Konstitution von Märkten. Wiesbaden: Westdeutscher Verlag.

Dahl, Robert A. 1971. Polyarchy: Participation and Opposition. New Haven: Yale University Press. 
Debus, Marc. 2007. Bestimmungsfaktoren des Wahlverhaltens in Deutschland bei den Bundestagswahlen 1987, 1998 und 2002: Eine Anwendung des Modells von Adams, Merrill und Grofman. Politische Vierteljahresschrift 48: 269-292.

Debus, Marc. 2009. Pre-electoral Commitments and Government Formation. Public Choice 138: 45-64.

De Swan, Abram. 1973. Coalition Theories and Cabinet Formations. A Study of Formal Theories of Coalition Formation Applied to Nine European Parliaments after 1918. Amsterdam u. a.: Elsevier/Jossey Bass.

Diermeier, Daniel. 1996. Rational Choice and the Role of Theory in Political Science. In: Jeffrey Friedmann (Hrsg.), The Rational Choice Controversy. Economic Models of Politics Reconsidered. New Haven: Yale University Press, 59-71.

Döhler, Marian. 1990. Gesundheitspolitik nach der ,Wende“. Policy-Netzwerke und ordnungspolitischer Strategiewechsel in Großbritannien, den USA und der Bundesrepublik Deutschland. Berlin: edition sigma.

Döhler, Marian und Philip Manow. 1997. Strukturbildung von Politikfeldern - Das Beispiel bundesdeutscher Gesundheitspolitik seit den 50er Jahren. Opladen: Leske + Budrich.

Döring, Herbert. 1995. Parliaments and Majority Rule in Western Europe. Frankfurt a.M./New York: Campus.

Döring, Herbert und Mark Hallerberg (Hrsg.). 2004. Patterns of Parliamentary Behaviour: Passage of Legislation Across Western Europe. London: Ashgate.

Dos Santos, Theotonio. 1969. La crisis de la teoria del desarrollo y las relaciones de dependencia en América Latina. Santiago de Chile: Siglo 21.

Dowding, Keith. 2000. Institutionalist Research on the European Union: A Critical Review. European Union Politics 1: 125-144.

Downs, Anthony. 1957. An Economic Theory of Democracy. New York: Harper.

Downs, Anthony. 1967. Inside Bureaucracy. Boston: Little, Brown \& Company.

Druwe, Ulrich und Volker Kunz (Hrsg.). 1994. Rational Choice in der Politikwissenschaft. Grundlagen und Anwendungen. Opladen: Leske + Budrich.

Druwe, Ulrich und Volker Kunz (Hrsg.). 1996. Handlungs- und Entscheidungstheorie in der Politikwissenschaft. Eine Einführung in Konzepte und Forschungsstand. Opladen: Leske + Budrich.

Druwe, Ulrich und Volker Kunz (Hrsg.). 1998. Anomalien in Handlungs- und Entscheidungstheorien. Opladen: Leske + Budrich.

Druwe, Ulrich und Volker Kunz (Hrsg.). 1999. Politische Gerechtigkeit. Opladen: Leske + Budrich.

Dunleavy, Patrick. 1997. Explaining the Centralization of the European Union: A Public Choice Analysis. Aussenwirtschaft 52: 183-212.

Dylla, Daria W. 2007. Der Rational-Choice Ansatz und die Ursache politischer Entscheidungen. Zeitschrift für Politikwissenschaft 17: 1163-1184.

Esping-Andersen, Gøsta. 1990. The Three Worlds of Welfare Capitalism. Princeton: Princeton University Press.

Falter, Jürgen und Michèle Knodt. 2007. Die Bedeutung von Themenfeldern, theoretischen Ansätzen und die Reputation von Fachvertretern. Politikwissenschaft. Rundbrief der Deutschen Vereinigung für Politische Wissenschaft 137: 147-160.

Fearon, James D. 1995. Rationalist Explanations for War. International Organizations 49: 379414.

Fearon, James D. und David Laitin. 1996. Explaining Interethnic Cooperation. American Political Science Review 90: 715-735.

Fearon, James D. und Alexander Wendt. 2002. Rationalism v. Constructivism: A Sceptical View. In: Walter Carlsnaess, Beth Simmons und Thomas Risse (Hrsg.), Handbook of International Relations. London: SAGE, 52-75. 
Fehr, Ernst und Simon Gächter. 2002. Altruistic Punishment in Humans. Nature 415: 137-140.

Fehr, Ernst, Herbert Gintis, Samuel Bowles und Robert Boyd. 2003. Explaining Altruistic Behaviour in Humans. Evolution and Human Behavior 24: 153-172.

Ferejohn, John. 1986. Incumbent Performance and Electoral Control. Public Choice 50: 5-25.

Finke, Daniel. 2009a. Challenges to Intergovernmentalism: An Empirical Analysis of EU Treaty Negotiations since Maastricht. West European Politics 32: 466-495.

Finke, Daniel. 2009b. Estimating the Effect of Non-Separable Preferences in EU Treaty Negotiations. Journal of Theoretical Politics [im Erscheinen].

Fiorina, Morris. 1976. The Voting Decision: Instrumental and Expressive Aspects. Journal of Politics 38: 390-413.

Franchino, Fabio. 2000. Commission's Executive Discretion: Information and Comitology. Journal of Theoretical Politics 12: 155-181.

Freitag, Markus und Adrian Vatter. 2007. The Contradictory Effects of Consensus Democracy on the Size of Government: Evidence from the Swiss Cantons. British Journal of Political Science 36: 359-371.

Frey, Bruno S. 1977. Moderne Politische Ökonomie. München: Piper.

Frey, Bruno S. 1981. Theorie demokratischer Wirtschaftspolitik. München: Vahlen.

Frey, Bruno S. 1990. Ökonomie ist Sozialwissenschaft. Die Anwendung der Ökonomie auf neue Gebiete. München: Vahlen.

Friedman, Jeffrey (Hrsg.). 1996. The Rational Choice Controversy. Economic Models of Politics Reconsidered. New Haven: Yale University Press.

Fuchs, Dieter und Steffen Kühnel. 1994. Wählen als rationales Handeln: Anmerkungen zum Nutzen des Rational-Choice-Ansatzes in der empirischen Wahlforschung. In: Hans-Dieter Klingemann und Max Kaase (Hrsg.), Wahlen und Wähler - Analysen aus Anlass der Bundestagswahl 1990. Opladen: Westdeutscher Verlag, 305-364.

Fudenberg, Drew und Jean Tirole. 1991. Game Theory. Cambridge: MIT-Press.

Füss, Roland und Michael M. Bechtel. 2008. Partisan Politics and Stock Market Performance: The Effect of Expected Government Partisanship on Stock Returns in the 2002 Federal Election. Public Choice 135: 131-150.

Galtung, Johan. 1971. A Structural Theory of Imperialism. Journal of Peace Research 8: 81117.

Ganghof, Steffen. 2003. Promises and Pitfalls of Veto Player Analysis. Swiss Political Science Review 9: 1-22.

Ganghof, Steffen. 2005. Politische Gleichheit und echte Mehrheitsdemokratie. Über die normativen Grundlagen institutioneller Arrangements. Zeitschrift für Politikwissenschaft 15: 741763.

Ganghof, Steffen. 2006. The Politics of Income Taxation. A Comparative Analysis. Colchester: ECPR Press.

Ganghof, Steffen und Philip Manow (Hrsg.). 2005. Mechanismen der Politik. Strategische Interaktion im deutschen Regierungssystem. Frankfurt a. M.: Campus.

Ganghof, Steffen, Christoph Hönnige und Christian Stecker (Hrsg.). 2009. Parlamente, Agendasetzung und Vetospieler. Festschrift für Herbert Döring. Wiesbaden: VS Verlag für Sozialwissenschaften.

Garrett, Geoffrey. 1992. International Cooperation and Institutional Choice: The European Community's Internal Market. International Organization 46: 533-560.

Garrett, Geoffrey und George Tsebelis. 1996. The Limitations of Intergovernmentalism: An Institutional Critique. International Organization 50: 269-99.

Garrett, Geoffrey und Barry R. Weingast. 1993. Ideas, Interests, and Institutions: Constructing the European Community's Internal Market. In: Judith Goldstein und Robert O. Keohane (Hrsg.), Ideas and Foreign Policy. Beliefs, Institutions, and Political Change. Ithaca: Cornell University Press, 173-206. 
Genschel, Philipp. 2002. Steuerwettbewerb und Steuerharmonisierung in der Europäischen Union. Frankfurt a. M.: Campus.

Genschel, Philipp und Thomas Plümper. 1997. Regulatory Competition and International Cooperation. Journal of European Public Policy 4: 626-642.

Genschel, Philipp und Klaus Schlichte. 1997. Wenn Kriege chronisch werden: Der Bürgerkrieg. Leviathan 25: 501-517.

Genschel, Philipp und Bernhard Zangl. 2008. Metamorphosen des Staates - vom Herrschaftsmonopolisten zum Herrschaftsmanager. Leviathan 36: 430-454.

Goodin, Robert E., James M. Rice, Antti Parpo and Lina Eriksson. 2008. Discretionary Time: A New Measure of Freedom. Cambridge: Cambridge University Press.

Green, David P. und Ian Shapiro. 1994. Pathologies of Rational Choice Theory: A Critique of Applications in Political Science. New Haven: Yale University Press.

Greif, Avner. 1993. Contract Enforceability and Economic Institutions in Early Trade: The Maghribi Traders' Coalition. American Economic Review 83: 525-48.

Grobe, Christian. 2007. Kooperation und Verhandlungen in den Internationalen Beziehungen. Eine Neubetrachtung der ZIB-Debatte aus rationalistischer Perspektive. Marburg: Tectum.

Grofman, Bernard. 2004a. Reflections on Public Choice. Public Choice 118: 31-51.

Grofman, Bernard. 2004b. Downs and Two-Party Convergence. Annual Review of Political Science 7: $25-46$

Güth, Werner, Rolf Schmittberger und Bernd Schwarze. 1982. An Experimental Analysis of U1timatum Bargaining. Journal of Economic Behavior and Organization 3: 367-388.

Güth, Werner, Sven Fischer, Andreas Stiehler und Wieland Müller. 2006. From Ultimatum to Nash Bargaining: Theory and Experimental Evidence. Experimental Economics 9: 17-33.

Hättich, Manfred. 1966. Begriff und Formen der Demokratie. Mainz: Hase \& Koehler.

Hanrieder, Tine. 2008. Moralische Argumente in den Internationalen Beziehungen. Zeitschrift für Internationale Beziehungen 15: 161-186.

Hainmüller, Jens, Holger Lutz Kern und Michael Bechtel. 2006. Wahlkreisarbeit zahlt sich doppelt aus - Zur Wirkung des Amtsinhaberstatus einer Partei auf ihren Zweitstimmenanteil bei den Bundestagswahlen 1949-1998. Jahrbuch für Handlungs- und Entscheidungstheorie 4. Wiesbaden: VS Verlag für Sozialwissenschaften, 11-45.

Hall, Peter A. und David Soskice. 2001. Varieties of Capitalism: The Institutional Foundations of Comparative Advantage. Oxford: Oxford University Press.

Henning, Christian H. C. A. 2000. Macht und Tausch in der europäischen Agrarpolitik: eine positive Theorie kollektiver Entscheidungen. Frankfurt a. M.: Campus.

Heritiér, Adrienne. 2002. Common Goods: Reinventing European and International Governance. Lanham: Rowman \& Littlefield.

Herrmann, Michael, Susumu Shikano, Paul W. Thurner und Axel Becker. 2006. Die Analyse von Wählerpräferenzen mit Rank Ordered Logit. Jahrbuch für Handlungs- und Entscheidungstheorie 4. Wiesbaden: VS Verlag für Sozialwissenschaften, 113-134.

Herne, Kaisa und Hannu J. Nurmi. 1993. The Distribution of A Priori Voting Power in the EC Council of Ministers and the European Parliament. Scandinavian Political Studies 16: 269284

Hirschmann, Albert O. 1970. Exit, Voice and Loyalty: Responses in Decline in Firms, Organizations and States. Cambridge: Harvard University Press.

Hirschmann, Albert O. 1992. Abwanderung, Widerspruch und das Schicksal der Deutschen Demokratischen Republik. Leviathan 20: 330-358.

Hirschmann, Albert O. 1994. Wieviel Gemeinsinn braucht die liberale Gesellschaft? Leviathan 22: $293-304$

Hirschmann, Albert O. 1995. Grenzübertritte. Orte und Ideen eines Lebenslaufes. Leviathan 23: 263-304 
Hix, Simon. 1996. The European Party Federations: From Transnational Party Cooperation to Nascent European Parties. In: John Gaffney (Hrsg.), Political Parties and the European Union. London: Routledge, 308-331.

Hix, Simon. 1998. Elections, Parties and Institutional Designs. A Comparative Perspective on European Union Democracy. West European Politics 21: 19-52.

Hönnige, Christoph. 2007. Verfassungsgericht, Regierung und Opposition. Die vergleichende Analyse eines Spannungsdreiecks. Wiesbaden: VS Verlag für Sozialwissenschaften.

Holler, Manfred J. (Hrsg.). 1981. Power, Voting and Voting Power. Würzburg: Physica.

Holzinger, Katharina. 1987. Umweltpolitische Instrumente aus der Sicht der staatlichen Bürokratie. München: Ifo-Institut für Wirtschaftsforschung.

Holzinger, Katharina. 1994. Politik des kleinsten gemeinsamen Nenners? Berlin: edition sigma.

Holzinger, Katharina. 2001. Kommunikationsmodi und Handlungstypen in den Internationalen Beziehungen. Anmerkungen zu einigen irreführenden Dichotomien. Zeitschrift für Internationale Beziehungen 8: 243-286.

Holzinger, Katharina. 2008. Transnational Common Goods: Strategic Constellations, Collective Action Problems, and Multi-Level Provision. New York: Palgrave Macmillan.

Holzinger, Katharina und Christoph Knill. 2001. Institutionelle Entwicklungspfade im europäischen Integrationsprozess - Eine konstruktive Kritik an Joschka Fischers Vorschlägen. Zeitschrift für Politikwissenschaft 11: 987-1010.

Holzinger, Katharina, Christoph Knill und Helge Jörgens. 2007. Transfer, Diffusion und Konvergenz von Politiken. PVS-Sonderheft 38. Wiesbaden: VS Verlag für Sozialwissenschaften.

Homann, Karl. 1988. Rationalität und Demokratie. Tübingen: Mohr.

Höpner, Martin. 2007. Ist Politik gegen Verbände möglich? 25 Jahre Mancur Olsons „The Rise and Decline of Nations“. Leviathan 35: 310-347.

Horbach, Jens. 1992. Neue Politische Ökonomie und Umweltpolitik. Frankfurt a.M./New York: Campus.

Hosli, Madeleine O. 1996. Coalitions and Power: Effects of Qualified Majority Voting in the Council of the European Union. Journal of Common Market Studies 34: 255-273.

Hosli, Madeleine O. 1997. Voting Strength in the European Parliament. European Journal of Political Research 31: 351-366.

Hug, Simon und Thomas König. 2007. Domestic Structures and Constitution-Building in an International Organization. The Review of International Organizations, Special Issue 2: 105218.

Immergut, Ellen. 1990. Institutions, Veto Points, and Policy Results: A Comparative Analysis of Health Care. Journal of Public Policy 10: 391-416.

Jepperson, Ronald J., Alexander Wendt und Peter J. Katzenstein. 1996. Norms, Identity, and Culture in National Security. In: Peter J. Katzenstein (Hrsg.), The Culture of National Security. New York: Columbia University Press, 33-75.

Jordana, Jacint. 1992. Interessenkonflikte und institutioneller Kontext in der spanischen Gewerkschaftspolitik. Eine spieltheoretische Interpretation. Politische Vierteljahresschrift 33: $73-$ 92.

Junge, Dirk und Thomas König. 2007. What's Wrong with EU Spatial Analysis? The Accuracy and Robustness of Empirical Applications in Light of Process Interpretation and Preference Specification. Journal of Theoretical Politics 19: 465-487.

Kagel, John H. und Alvin E. Roth. 1995. The Handbook of Experimental Economics. Princeton: Princeton University Press.

Kahler, Miles. 1998. Rationality in International Relations. International Organization 52: 919941.

Kahnemann, Daniel, Amos Tversky und Paul Slovic (Hrsg.). 1982. Judgment under Uncertainty: Heuristics and Biases. Cambridge: Cambridge University Press.

Kaiser, Andre. 2002. Mehrheitsdemokratie und Institutionenreform. Frankfurt a. M.: Campus. 
Kappelhoff, Peter und Franz U. Pappi. 1984. Abhängigkeit, Tausch und kollektive Entscheidungen in einer Gemeindeelite. Zeitschrift für Soziologie 13: 87-117.

Kasack, Christiane. 2004. The Legislative Impact of the European Parliament Under the Revised Co-Decision Procedure. European Union Politics 5: 241-260.

Keck, Otto. 1984. Der Schnelle Brüter: Ein Lehrstück für die Technologiepolitik. Politische Vierteljahresschrift 25: 296-315.

Keck, Otto. 1991. Der neue Institutionalismus in der Theorie der Internationalen Politik. Politische Vierteljahresschrift 32: 635-653.

Keck, Otto. 1995. Rationales kommunikatives Handeln in den internationalen Beziehungen. Ist eine Verbindung von Rational-Choice-Theorie und Habermas' Theorie des kommunikativen Handelns möglich? Zeitschrift für Internationale Beziehungen 2: 5-48.

Keller, Berndt. 1988. Olsons „Logik des kollektiven Handelns“. Entwicklung, Kritik - und eine Alternative. Politische Vierteljahresschrift 29: 388-406.

Kemmerling, Achim und Andreas Stephan. 2002. The Contribution of Local Public Infrastructure to Private Productivity and Its Political Economy: Evidence from a Panel of Large German Cities. Public Choice 113: 403-424.

Keohane, Robert O. und Helen V. Milner. 1996. Internationalization and Domestic Politics. New York: Cambridge University Press.

Kern, Lucian. 1979. Sind demokratische Entscheidungsregeln verfälschungsfrei? Eine politische Interpretation einiger Ergebnisse der Theorie kollektiver Entscheidungen. Politische Vierteljahresschrift 20: 330-356.

Kern, Lucian und Hans Peter Müller. 1986. Gerechtigkeit, Diskurs oder Markt? Die neuen Ansätze in der Vertragstheorie. Opladen: Westdeutscher Verlag.

Kirchgässner, Gebhard. 1977. Wirtschaftslage und Wählerverhalten. Eine empirische Studie für die Bundesrepublik Deutschland von 1971 bis 1976. Politische Vierteljahresschrift 18: 510536.

Kirchgässner, Gebhard. 1991. Homo oeconomicus. Tübingen: Mohr.

Kirsch, Guy. 1974. Ökonomische Theorien der Politik. Düsseldorf: Werner.

Kirsch, Guy. 2004. Neue Politische Ökonomie. Stuttgart: Lucius \& Lucius.

Kliemt, Hartmut. 1984. Nicht-explanative Funktionen eines „Homo oeconomicus“ und Beschränkungen seiner explanativen Rolle. Homo oeconomicus 2: 7-49.

König, Thomas. 1992. Kollektive Entscheidungsfindung im deutschen Politikfeld „Arbeit“: Ein modellorientierter Politikfeldansatz. Politische Vierteljahresschrift 33: 597-621.

König, Thomas. 1996. The Constitutional Development of European Integration. Journal of Theoretical Politics 8: 553-559.

König, Thomas. 1997. Politikverflechtungsfalle oder Parteienblockade? Das Potential für politischen Wandel im deutschen Zweikammersystem. Zeitschrift für Staatswissenschaft und Staatspraxis 2:135-159.

König, Thomas. 2005. Measuring and Analysing Positions on European Constitution-building. European Union Politics 6: 259-267.

König, Thomas. 2008. Analysing the Process of EU Legislative Decision-Making: To Make a Long Story Short... European Union Politics 9: 145-165.

König, Thomas und Thomas Bräuninger. 1997. Wie wichtig sind die Länder für die Politik bei Einspruchs- und Zustimmungsgesetzen? Zeitschrift für Parlamentsfragen 28: 605-628.

König, Thomas und Thomas Bräuninger. 1999. Finnland auf dem Weg zum Mehrheitssystem? Eine vergleichende Analyse der politischen Willensbildung vor und nach den finnischen Verfassungsreformen der 90er Jahre. Politische Vierteljahresschrift 40: 40-64.

König, Thomas und Thomas Bräuninger. 2000. Decisiveness and Inclusiveness: Two Aspects of the Intergovernmental Choice of European Voting Rules. Homo Oeconomicus 17: 107-123.

König, Thomas und Thomas Bräuninger. 2004. Accession and Reform of the European Union. A Game-theoretical Analysis of Eastern Enlargement and the Constitutional Reform. European Union Politics 5: 419-439. 
König, Thomas, Stephanie Daimer und Daniel Finke. 2008. The Treaty Reform of the EU: Constitutional Agenda-Setting. Journal of Common Market Studies 46: 337-363.

König, Thomas und Daniel Finke. 2007. Reforming the Equilibrium? Veto Players and Policy Change in the European Constitution-building Process. Review of International Organizations 2: 153-176.

König, Thomas und Mirja Pöter. 2001. Examining the EU Legislative Process. The Relative Importance of Agenda and Veto Power. European Union Politics 2: 329-351.

König, Thomas und Sven-Oliver Proksch. 2006. Exchanging and Voting in the Council: Endogenizing the Spatial Model of Legislative Politics. Journal of European Public Policy 13: 647-669.

König, Thomas und Jonathan Slapin. 2006. From Unanimity to Consensus. An Analysis of the Negotiation at the EU's Constitutional Convention. World Politics 58: 413-445.

König, Thomas und Vera Troeger. 2005. Budgetary Politics and Veto Players. Swiss Political Science Review 11: 47-75.

Kroneberg, Clemens. 2006. Die Erklärung der Wahlteilnahme und die Grenzen des RationalChoice-Ansatzes. Eine Anwendung des Modells der Frame-Selection. Jahrbuch für Handlungs- und Entscheidungstheorie 4, Wiesbaden: VS Verlag für Sozialwissenschaften, 79111.

Krueger, Anne. 1974. The Political Economy of the Rent-Seeking Society. American Economic Review 64: 291-303.

Kunz, Volker. 1996. Empirische Ökonomik. Handlungstheoretische Grundlagen der Erklärung politischer und sozialer Prozesse. Marburg: Metropolis.

Kunz, Volker. 1997. Theorie rationalen Handelns. Konzepte und Anwendungsprobleme. Opladen: Leske + Budrich.

Kunz, Volker. 2004. Rational Choice. Frankfurt a. M.: Campus.

Lane, Jan-Erik und Reinert Maeland. 1995. Voting Power Under the EU Constitution. Journal of Theoretical Politics 7: 223-230.

Laver, Michael J. 1997. Government Formation in the European Parliament. Aussenwirtschaft 52: 223-248.

Laver, Michael J. und Kenneth A. Shepsle. 1996. Making and Breaking Governments. Cambridge: Cambridge University Press.

Lehner, Franz. 1981. Einführung in die NPÖ. Königstein: Athenäum.

Lehner, Franz, Klaus Schubert und Birgit Geile. 1983. Die strukturelle Rationalität regulativer Wirtschaftspolitik. Politische Vierteljahresschrift 24: 361-384.

Lijphart, Arend. 1992. Parliamentary versus Presidential Government. Oxford: Oxford University Press.

Lijphart, Arend. 1999. Patterns of Democracy: Government Forms and Performance in Thirty-Six Countries. New Haven: Yale University Press.

Linhart, Eric und Franz U. Pappi. 2009. Koalitionsbildungen zwischen Ämter- und Politikmotivation. Konstruktion einer interdependenten Nutzenfunktion. Politische Vierteljahresschrift 50: 23-49.

Linhart, Eric, Franz U. Pappi und Ralf Schmitt. 2008. Die proportionale Ministerienaufteilung in deutschen Koalitionsregierungen: akzeptierte Norm oder das Ausnutzen strategischer Vorteile? Politische Vierteljahresschrift 49: 46-67.

Lipset, Seymour M. 1959. Some Social Requisites of Democracy: Economic Development and Political Legitimacy. American Political Science Review 53: 69-105.

List, Christian. 2004. Multidimensional Welfare Aggregation. Public Choice 119: 119-142.

Lohmann, Susanne. 1996. The Poverty of Green and Shapiro. In: Jeffrey Friedmann (Hrsg.), The Rational Choice Controversy. Economic Models of Politics Reconsidered. New Haven: Yale University Press, 127-155. 
Lütz, Susanne. 2002. Der Staat und die Globalisierung von Finanzmärkten. Frankfurt a. M./New York: Campus.

Lütz, Susanne und Roland Czada (Hrsg.) 2004. Der Wohlfahrtsstaat - Transformation und Perspektiven. Wiesbaden: VS Verlag für Sozialwissenschaften.

Mahoney, James. 2000. Rational Choice Theory and the Comparative Method: An Emerging Synthesis? Studies in Comparative International Development 35: 83-94.

Maloy, Jason S. 2008. An Genealogy of Rational Choice: Rationalism, Elitism, and Democracy. Canadian Journal of Political Science 41: 749-771.

Mann, Irwin und Lloyd S. Shapley. 1964. The A Priori Voting Strength of the Electoral College. In: Martin Shubik (Hrsg.), Game Theory and Related Approaches to Social Behaviour. New York u. a.: Wiley, 151-164.

Manow, Philip. 2002. Was erklärt politische Patronage in den Ländern Westeuropas? Defizite des politischen Wettbewerbs oder historisch-formative Phasen der Massendemokratisierung. Politische Vierteljahresschrift 43: 20-45.

Manow, Philip. 2003. Low Trust and High Trust Equilibria in Politics - Political Corruption and Party Patronage as Coordination Games. In: Thomas Plümper und Hans-Peter Burth (Hrsg.), Jahrbuch für Entscheidungs- und Handlungstheorie. Opladen: Leske + Budrich, 7-31.

Manow, Philip. 2004. Der demokratische Leviathan - eine kurze Geschichte parlamentarischer Sitzanordnungen seit der französischen Revolution. Leviathan 32: 319-347.

Manow, Philip. 2006. Die politische Anatomie demokratischer Repräsentation. Leviathan 34: 149-181.

Manow, Philip. 2007. Sexualität und Souveränität - Neue Nachrichten vom Vor- und Nachleben des Leviathan-Frontispizes. Leviathan 35: 470-494.

Marsh, David und Heather Savigny. 2004. Political Science as a Broad Church: The Search for a Pluralist Discipline. Politics 24: 155-68.

Martin, Christian W. 2004. Demokratie, Autokratie und die regulative Gestaltung der Außenwirtschaftsbeziehungen in Entwicklungsländern. Politische Vierteljahresschrift 45: 32-54.

Martin, Christian W. 2005. Die doppelte Transformation: Demokratie und Außenwirtschaftsliberalisierung in Entwicklungsländern. Wiesbaden: VS Verlag für Sozialwissenschaften.

Martin, Christian und Thomas Plümper. 2006. Instabilität von parteipolitischen Programmen: Der Einfluss der Zahl der Parteien. Jahrbuch für Handlungs- und Entscheidungstheorie 4. Wiesbaden: VS Verlag für Sozialwissenschaften, 135-150.

Marx, Johannes. 2006. Vielfalt oder Einheit in den Theorien der Internationalen Beziehungen. Baden-Baden. Nomos.

Mayntz, Renate und Fritz W. Scharpf (Hrsg.). 1995. Gesellschaftliche Selbstregelung und politische Steuerung. Frankfurt a. M.: Campus.

McCarthy, Nolan und Adam Meirowitz. 2007. Political Game Theory. An Introduction. Cambridge: Cambridge University Press.

McCubbins, Mathew D. und Talbot Page. 1987. A Theory of Congressional Delegation. In: McCubbins, Mathew D. und Terry Sullivan (Hrsg.), Congress: Structure and Policy. New York: Cambridge University Press, 409-425.

McKelvey, Richard D. 1976. Intransitives in Multidimensional Voting Models and Some Implications for Agenda Control. Journal of Economic Theory 12: 472-482.

McKelvey, Richard D. und Norman Schofield. 1986. Structural Instability of the Core. Journal of Mathematical Economics 15: 179-198.

McLean, Iain. 1987. Public Choice. An Introduction. Oxford: Blackwell.

Mensch, Kirsten. 1996. Internalistische versus externalistische Erklärungsprinzipien in RationalChoice-Ansätzen oder: Wie erklärt die Rational-Choice-Theorie die Höhe der Wahlbeteiligung? Politische Vierteljahresschrift 37: 80-99.

Migué, Jean-Luc und Gerard Bélanger. 1974. Toward a General Theory of Managerial Discretion. Public Choice 17: 27-43. 
Mitchell, William C. 1999. Political Science and Public Choice: 1950-1970. Public Choice 98: 237-249.

Morgenthau, Hans J. 1948. Politics Among Nations. The Struggle for Power and Peace. New York: Alfred A. Knopf.

Morrow, James D. 1994. Game Theory for Political Scientists. Princeton: Princeton University Press.

Moser, Peter, Gerald Schneider und Gebhard Kirchgässner (Hrsg.). 1997. Decision Rules in the European Union: A Rational Choice Perspective. Aussenwirtschaft 52, Special Issue.

Mueller, Dennis C. 2001. Public Choice after 50 Years, Bruno Frey after 60. Kyklos 54: 343354.

Mueller, Dennis C. 2003. Public Choice III. Cambridge: Cambridge University Press.

Müller, Harald. 1994. Internationale Politik als kommunikatives Handeln. Zur Kritik der utilitaristischen Handlungstheorien. Zeitschrift für Internationale Beziehungen 1: 15-44.

Müller, Harald. 1995. Spielen hilft nicht immer. Die Grenzen des Rational-Choice-Ansatzes und der Platz der Theorie kommunikativen Handelns in der Analyse internationaler Beziehungen. Zeitschrift für Internationale Beziehungen 2: 371-392.

Nash, John. 1950a. The Bargaining Problem. Econometrica 18: 155-162.

Nash, John. 1950b. Non-cooperative Games. Princeton: Princeton University Press.

von Neumann, John und Oskar Morgenstern. 1944. Theory of Games and Economic Behavior. Princeton: Princeton University Press.

Niskanen, William A. 1971. Bureaucracy and Representative Government. Chicago: AldineAtherton.

North, Douglass C. 1981. Structure and Change in Economic History. New York: Norton.

North, Douglass C. 1990. Institutions, Institutional Change and Economic Performance. Cambridge: Cambridge University Press.

Nurmi, Hanno. 1987. Comparing Voting Systems. Dordrecht: D. Reidel.

Nurmi, Hanno und Tommi Meskanen. 1999. A Priori Power Measures and the Institutions of the European Union. European Journal of Political Research 35: 161-179.

Obinger, Herbert, Uwe Wagschal und Bernhard Kittel (Hrsg.). 2003. Politische Ökonomie: Demokratie und wirtschaftliche Leistungsfähigkeit. Opladen: Leske + Budrich.

Ockenfels, Axel. 1999. Fairneß, Reziprozität und Eigennutz. Tübingen: Mohr.

Olson, Mancur. 1965. The Logic of Collective Action: Public Goods and the Theory of Groups. Cambridge: Harvard University Press.

Olson, Mancur. 1993. Dictatorship, Democracy and Development. American Political Science Review 87: 567-576.

Olson, Mancur. 2000. Power and Prosperity: Outgrowing Communist and Capitalist Dictatorships. New York: Oxford University Press.

Ordeshook, Peter C. 1986. Game Theory and Political Theory: An Introduction. New York: Cambridge University Press.

Ordeshook, Peter C. 1992. A Political Theory Primer. New York/London: Routledge.

Ostrom, Elinor. 1990. Governing the Commons: The Evolution of Institutions for Collective Action. New York: Cambridge University Press.

Ostrom, Elinor. 1998. A Behavioral Approach to the Rational Choice Theory of Collective Action. American Political Science Review 92: 1-22.

Ostrom, Elinor. 2005. Understanding Institutional Diversity. Princeton: Princeton University Press.

Pajala, Antti und Mika Widgrén. 2004. A Priori versus Empirical Voting Power in the EU Council of Ministers. European Union Politics 5: 73-97.

Pappi, Franz U. 1973. Parteiensystem und Sozialstruktur in der Bundesrepublik Deutschland. Politische Vierteljahresschrift 14: 191-213

Pappi, Franz U. 1987. Methoden der Netzwerkanalyse. München: Oldenbourg. 
Pappi, Franz U. und Christian Melbeck. 1984. Das Machtpotential von Organisationen in der Gemeindepolitik. Kölner Zeitschrift für Soziologie und Sozialpsychologie 36: 557-584.

Pappi, Franz U., Thomas König und David Knoke. 1995. Entscheidungsprozesse in der Arbeitsund Sozialpolitik. Der Zugang der Interessengruppen zum Regierungssystem über Politikfeldnetze: Ein deutsch-amerikanischer Vergleich. Frankfurt a. M./NewYork: Campus.

Pappi, Franz U. und Susumu Shikano. 2001. Personalisierung der Politik in Mehrparteiensystemen am Beispiel deutscher Bundestagswahlen seit 1980. Politische Vierteljahresschrift 42: 355-387.

Pappi, Franz U., Axel Becker und Alexander Herzog. 2005. Regierungsbildung in Mehrebenensystemen: Zur Erklärung der Koalitionsbildung in den deutschen Bundesländern. Politische Vierteljahresschrift 46: 432-458.

Pappi, Franz U. und Susumu Shikano. 2007. Wahl- und Wählerforschung. Baden-Baden: Nomos.

Persson, Torsten und Guido Tabellini. 2003. The Economic Effect of Constitutions. Cambridge: MIT-Press.

Pies, Ingo. 1993. Normative Institutionenökonomik. Tübingen: Mohr.

Plümper, Thomas. 2001. Die Politik wirtschaftlichen Wachstums in autoritären Staaten. Politische Vierteljahresschrift 42: 79-100.

Plümper, Thomas und Christian W. Martin. 2003. Democracy, Government Spending, and Economic Growth: A Political-Economic Explanation of the Barro-Effect. Public Choice 117: 27-50.

Polachek, Solomon. 1980. Conflict and Trade. Journal of Conflict Resolution 24: 55-78.

von Prittwitz, Volker. 1996. Verständigung über die Verständigung. Anmerkungen und Ergänzungen zur Debatte über Rationalität und Kommunikation in den Internationalen Beziehungen. Zeitschrift für Internationale Beziehungen 3: 133-148.

Quinn, Dennis. 1997. The Correlates of Change in International Financial Regulation. American Political Science Review 91: 531-551.

Rabushka, Alvin und Kenneth Shepsle. 1971. Political Entrepreneurship and Patterns of Democratic Instability in Plural Society. Race 12: 461-476.

Rapoport, Anatol. 1970. Über die Anwendbarkeit von Friedensforschung. Politische Vierteljahresschrift 11: 518-531

Rattinger, Hans und Walter Puschner. 1981. Ökonomie und Politik in der Bundesrepublik. Wirtschaftslage und Wahlverhalten 1953-1980. Politische Vierteljahresschrift 22: 264-286.

Rawls, John. 1971. A Theory of Justice. Cambridge: Belknap Press.

Riker, William H. 1963. The Theory of Political Coalitions. New Haven: Yale University Press.

Riker, William H. 1993. Agenda Formation. Ann Arbor: University of Michigan Press.

Riker, William H. und L. S. Shapley. 1968. Weighted Voting: A Mathematical Analysis for Instrumental Judgements. In: J. Ronald Pennock und John W. Chapman (Hrsg.), Nomos X: Representation. New York: Atherton Press, 199-216.

Risse-Kappen, Thomas. 1995. Reden ist nicht billig. Zur Debatte um Kommunikation und Rationalität. Zeitschrift für Internationale Beziehungen 2: 171-184.

Rodrik, Dani. 1998. Why Do Open Countries Have Bigger Governments? Journal of Political Economy 106: 997-1032.

Rogowski, Ronald. 1987. Political Cleavages and Changing Exposure to Trade. American Political Science Review 81: 1121-1137.

Sala-i-Martin, Xavier. 2006. The World Distribution of Income: Falling Poverty and Convergence. Quarterly Journal of Economics 121: 351-397.

Sattler, Thomas und Stefanie Walter. 2008. Wirtschaftspolitischer Handlungsspielraum im Zeitalter der Globalisierung. Eine empirische Untersuchung am Beispiel von Währungskrisen. Politische Vierteljahresschrift 49: 464-490 
Schäfer, Gregor. 2007. Spieltheorie und kommunikatives Handeln in den internationalen Beziehungen: eine Analyse der ZIB-Debatte (1994-2001). Marburg: Tectum.

Scharpf, Fritz W. 1985. Die Politikverflechtungs-Falle. Europäische Integration und deutscher Föderalismus im Vergleich. Politische Vierteljahresschrift 26: 323-356.

Scharpf, Fritz W. 1988. Inflation und Arbeitslosigkeit in Westeuropa. Eine spieltheoretische Interpretation. Politische Vierteljahresschrift 29: 6-41.

Scharpf, Fritz W. (Hrsg.). 1993. Games in Hierarchies and Networks. Analytical and Empirical Approaches to the Study of Governance Institutions. Frankfurt a. M.: Campus.

Scharpf, Fritz. 1997. Games Real Actors Play. Actor-Centered Institutionalism in Policy Research. Boulder/Oxford: Westview Press.

Scharpf, Fritz W. 2000. Interaktionsformen. Akteurzentrierter Institutionalismus in der Politikforschung. Wiesbaden: VS Verlag für Sozialwissenschaften.

Schimmelfennig, Frank. 1997. Rhetorisches Handeln in der internationalen Politik. Zeitschrift für Internationale Beziehungen 4: 219-254.

Schimmelfennig, Frank. 2004. Starke Anreize, ambivalente Wirkungen: Die Europäisierung Mittel- und Osteuropas. Leviathan 32: 250-268.

Schmalz-Bruns, Rainer. 1995. Die Theorie kommunikativen Handelns - eine Flaschenpost? Anmerkungen zur jüngsten Theoriedebatte in den Internationalen Beziehungen. Zeitschrift für Internationale Beziehungen 2: 347-370.

Schmidt, Manfred G. 2002. Political Performance and Types of Democracy. Findings from Comparative Research. European Journal of Political Research 41: 147-163.

Schmidt, Susanne K. und Raymund Werle. 1998. Coordinating Technology. Studies in the International Standardization of Telecommunications. Cambridge/London: MIT-Press.

Schneider, Christina J. 2007. Enlargement Processes and Distributional Conflicts: The Politics of Discriminatory Membership in the European Union. Public Choice 132: 85-102.

Schneider, Gerald. 1994. Rational Choice und kommunikatives Handeln. Eine Replik auf Harald Müller. Zeitschrift für Internationale Beziehungen 1: 357-366.

Schneider, Gerald. 1995. The Limits of Self-Reform: Institutional Building in the European Community. European Journal of International Relations 1: 59-86.

Schneider, Gerald und Claudia Seybold. 1997. Twelve Tongues, Once Voice: An Evaluation of European Political Cooperation. European Journal of Political Research 37: 367-396.

Schneider, Gerald und Stephanie Bailer. 2000. The Power of Legislative Hot Air. Informal Rules and the Enlargement Debate in the European Parliament. Journal of Legislative Studies 6: $19-44$.

Schneider, Gerald und Konstantin Baltz. 2003. The Power of Specialization: How Interest Groups Influence EU Legislation. Rivista di Politica Economica 93: 253-83.

Schneider, Volker. 1989. Technikentwicklung zwischen Politik und Markt. Der Fall Bildschirmtext. Frankfurt a. M.: Campus.

Schneider, Volker. 1999. Staat und Technische Kommunikation. Wiesbaden: Westdeutscher Verlag.

Schneider, Volker und Frank Janning. 2006. Politikfeldanalyse. Akteure, Diskurse und Netzwerke in der öffentlichen Politik. Wiesbaden: VS Verlag für Sozialwissenschaften.

Schultz, Kenneth A. 2001. Democracy and Coercive Diplomacy. Cambridge: Cambridge University Press.

Schulz, Heiner und Thomas König. 2000. Institutional Reform and Decision-Making. Efficiency in the European Union. American Journal of Political Science 44: 653-666.

Selb, Peter. 2008. Supersized Votes: Ballot Length, Uncertainty, and Choice in Direct Legislation Elections. Public Choice 135: 319-336.

Selten, Reinhard, Klaus Abbink und Ricarda Cox. 2005. Learning Direction Theory and the Winner's Curse. Experimental Economics 8: 5-20.

Shepsle, Kenneth. 1979. Institutional Arrangements and Equilibrium in Multidimensional Voting Models. American Journal of Political Science 23: 23-57. 
Shepsle, Kenneth and Barry Weingast. 1984. Uncovered Sets and Sophisticated Voting Outcomes with Implications for Agenda Institutions. American Journal of Political Science 25: 4975.

Shikano, Susumu. 2006. Modellgestützte Rekonstruktion und Simulation des Ergebnisses der Bundestagswahl 2005. In: Thomas Bräuninger und Joachim Behnke (Hrsg.), Jahrbuch für Handlungs- und Entscheidungstheorie 4. Opladen: VS Verlag für Sozialwissenschaften, 4778.

Shikano, Susumu, Bräuninger, Thomas und Joachim Behnke. 2009. Theorien der Verfassungsreform. Jahrbuch für Handlungs- und Entscheidungstheorie 5. Wiesbaden: VS Verlag für Sozialwissenschaften.

Shubik, Martin. 1982. Game Theory in the Social Sciences. Volume I and II. Cambridge: MITPress.

Simon, Herbert A. 1957. Models of Man. New York: Wiley.

Singer, David. 1969. Rückkoppelungsprozesse in Internationalen Konflikten. Politische Vierteljahresschrift 10: 2-20.

Slaughter, Anne-Marie. 1993. New Directions in Legal Research on the European Community. Journal of Common Market Studies 31: 391-400.

Small, Melvin und David J. Singer. 1976. The War Proneness of Democratic Regimes, 1816-1965. Jerusalem Journal of International Relations 1: 50-69.

Smith, Alastair. 1996. Endogenous Election Timing in Majoritarian Parliamentary System. Economics and Politics 8: 85-110.

Steffani, Winfried 1979: Parlamentarische und präsidentielle Demokratie. Strukutrelle Aspekte westlicher Demokratien. Opladen: Westdeutscher Verlag.

Steunenberg, Bernd. 1994. Decision-making under Different Institutional Arrangements: Legislation by the European Community. Journal of Theoretical and Institutional Economics 150: 642-669.

Strom, Kaare. 1990. Minority Government and Majority Rule. Cambridge: Cambridge University Press.

Strulik, Holger. 2007. A Distributional Theory of Government Growth. Public Choice 132: 305318 .

Taylor, Michael. 1987. The Possibility of Cooperation. Cambridge u.a.: Cambridge University Press.

Thurner, Paul W. 2006. Die graduelle Konstitutionalisierung der Europäischen Union. Tübingen: Mohr.

Thurner, Paul W. und Angelika Eymann. 2000. Policy-Specific Alienation and Indifference in the Calculus of Voting: A Simultaneous Model of Party Choice and Abstention. Public Choice 102: 51-77.

Thurner, Paul W. und Franz U. Pappi 2009. European Union Intergovernmental Conferences: Domestic Preference Formation, Transgovernmental Networks, and the Dynamics of Compromise. New York/London: Routledge.

Thurner, Paul W. und Michael Stoiber. 2002. Interministerielle Netzwerke: Formale und informelle Koordinationsstrukturen bei der Vorbereitung der deutschen Verhandlungsposition zur Regierungskonferenz 1996. Politische Vierteljahresschrift 43: 561-605.

Thurner, Paul W., Michael Stoiber und Cornelia Weinmann. 2005. Informelle transgouvernementale Koordinationsnetzwerke der Ministerialbürokratie der EU-Mitgliedstaaten bei einer Regierungskonferenz. Politische Vierteljahresschrift 46: 552-574.

Tullock, Gordon. 1987. Autocracy. Dordrecht: Kluwer.

Tsebelis, George. 1994. The Power of the European Parliament as a Conditional Agenda-Setter. American Political Science Review 88: 128-142.

Tsebelis, George. 1995. Conditional Agenda-Setting and Decision-making Inside the European Parliament. Journal of Legislative Studies 1: 65-93. 
Tsebelis, George. 1996. More on the European Parliament as a Conditional Agenda Setter. American Political Science Review 90: 839-844.

Tsebelis, George. 2000. Veto Players and Institutional Analysis. Governance 13: 441-474.

Tsebelis, George und Geoffrey Garret. 1997. Agenda Setting, Vetoes, and the EU's Codecision Procedure. Journal of Legislative Studies 3: 74-92.

Vatter, Adrian und Ruth Nabholz. 1995. Der Stimmbürger als Homo Oeconomicus? Ein empirischer Theorientest des Stimmbürgerverhaltens bei kantonalen Kreditvorlagen aus der Schweiz. Politische Vierteljahresschrift 36: 484-501.

Wagner, Harrison R. 2007. War and the State. Ann Arbor: University of Michigan Press.

Wagschal, Uwe, Thomas Metz und Nikolas Schwank. 2008. Was macht Staaten anfällig für Konflikte? Die Bedeutung demographischer Faktoren für inner- und zwischenstaatliche Konflikte. Zeitschrift für Politikwissenschaft 18: 353-383.

Waltz, Kenneth. 1979. Theory of International Politics. New York: McGraw-Hill.

Weede, Erich. 1985. Warum bleiben arme Leute arm? Rent-Seeking und Dependenz als Erklärungsansätze für die Armut in der Dritten Welt. Politische Vierteljahresschrift 26: 270-286.

Weiersmüller, Rudolf. 1971. Zur Machtverteilung in den Gremien der EWG. Schweizerische Zeitschrift für Volkswirtschaft und Statistik 107: 463-469.

Widgren Mika. 1994. Voting Power in the EC Decision Making and the Consequences of Two Different Enlargements. European Economic Review 38: 1153-1170.

Wiesenthal, Helmut. 1987. Rational Choice. Ein Überblick über Grundlinien, Theoriefelder und neuere Themenakquisition eines sozialwissenschaftlichen Paradigmas. Zeitschrift für Soziologie 16: 434-449.

Williamson, Oliver E. 1985. The Economic Institutions of Capitalism: Firms Markets, Relational Contracting. New York: Free Press.

Wintrobe, Ronald. 1998. The Political Economy of Dictatorship. Cambridge/New York: Cambridge University Press.

Zangl, Bernhard und Michael Zürn. 1996. Argumentatives Handeln bei internationalen Verhandlungen. Moderate Anmerkungen zur post-realistischen Debatte. Zeitschrift für Internationale Beziehungen 3: 341-366.

Zangl, Bernhard und Michael Zürn. 1999. Interessen in der internationalen Politik. Der akteursorientierte Institutionalismus als Brücke zwischen interesseorientierten und normorientierten Handlungstheorien. Zeitschrift für Politikwissenschaft 9: 923-950.

Zimmerling, Ruth. 1996. Wissenschaft und Verantwortung. Ist die traditionelle Gegenüberstellung von empirischer und normativer Politikwissenschaft haltbar? Zeitschrift für Politikwissenschaft 6: 51-82.

Zimmerling, Ruth. 2005. Influence and Power - Variations on a Messy Theme. Dordrecht: Springer.

Zintl, Reinhard. 1981. Politik aus ökonomischer Sicht. Zeitschrift für die gesamte Staatswissenschaft 137: 150-156.

Zintl, Reinhard. 1983. Individualistische Theorien und die Ordnung der Gesellschaft. Untersuchungen zur politischen Theorie von J. M. Buchanan und F. A. v. Hayek. Berlin: Duncker und Humblot.

Zintl, Reinhard. 1984. Erübrigen „ökonomische“ Verfassungstheorien die normative Diskussion über Entscheidungsregeln? In: Karl Peter Markl (Hrsg.), Analytische Politikphilosophie und ökonomische Rationalität, Band 2, Opladen: Westdeutscher Verlag, S. 27-40.

Zintl, Reinhard. 1986. Verfassungsvertrag oder Evolution - zu einer Kontroverse unter liberalen Theoretikern. Soziologische Revue 9: 249-255.

Zintl, Reinhard. 1989. Der Homo Oeconomicus: Ausnahmeerscheinung in jeder Situation oder Jedermann in Ausnahmesituationen? Analyse und Kritik 11: 52-69.

Zintl, Reinhard. 1994. Kooperation kollektiver Akteure - zum Informationsgehalt angewandter Spieltheorie. In: Julian Nida-Rümelin (Hrsg.), Praktische Rationalität. Grundlagenprobleme 
und ethische Anwendungen des Rational Choice-Paradigmas. Berlin/New York: De Gruyter, 239-258.

Zintl, Reinhard. 2000. Constitutions as External Constraints? In: Julian Nida-Rümelin und Wolfgang Spohn (Hrsg.), Rationality, Rules, and Structure. Dordrecht: Kluwer, 35-47.

Zohlnhöfer, Reimut. 2001. Die Bundesrepublik Deutschland im finanzpolitischen Zielkonflikt zwischen Wiedervereinigung und europäischer Integration. Zeitschrift für Politikwissenschaft 11: $1547-1571$

Zohlnhöfer, Reimut. 2009. Der Politikverflechtungsfalle entwischt? Die Effekte der Föderalismusreform I auf die Gesetzgebung. Zeitschrift für Politikwissenschaft 19: 39-76.

Zürn, Michael. 1992. Interessen und Institutionen in der internationalen Politik. Grundlegung und Anwendungen des situationsstrukturellen Ansatzes. Opladen: Leske + Budrich.

Zürn, Michael. 2003. US-Ordnung, Unordnung oder UN-Ordnung: Zur Entwicklung der Weltsicherheitsordnung. Leviathan 31: 441-449. 